\title{
Identification and Evaluation of High Yielding Good Quality Jack Genotypes
}

\author{
R. Jayavalli* \\ (Horticulture), Tamil Nadu Agricultural University, Agricultural College and \\ Research Institute, Kudumiyanmalai, India \\ *Corresponding author
}

\section{A B S T R A C T}

\begin{abstract}
The experiment was conducted with the bearing trees of different Jackfruit genotypes at Pudukkottai District of Tamil Nadu during the year 2016-2019. Fifty five jackfruit genotypes viz., KDM-AhJ-01 to KDM-AhJ-55 were selected for the study to identify superior ones. The age of the selected tress ranged from 07 to 33 years. The selected superior genotypes differ widely among themselves. Great variability exists with regards to many growth characters viz., tree height $2.20 \mathrm{~m}$ to $10.30 \mathrm{~m}$, trunk girth $(\mathrm{cm}) 45$ to $158 \mathrm{~cm}$, and canopy spread E-W direction $1.20-3.60 \mathrm{~m}$, canopy spread N-S direction 1.12 to $3.80 \mathrm{~m}$, month of flowering during November -December and January to February. Harvest during the month of January to March, April to June and July to September. Yield and quality characters viz., number of fruits per tree (10-50 nos.), individual fruit weight $(7-18 \mathrm{~kg})$, yield per tree $(80-810 \mathrm{~kg})$, fruit length $(11.00$ to $45.00 \mathrm{~cm}$ ), fruit breadth $(18.00$ to $57.00 \mathrm{~cm}$ ), TSS 6.00 to 29.00 ('brix), colour and appearance (4.00 to 9.00), flavor (4.00 to 9.00), fexture/firmness (4.00 to 9.00), taste (3.00 to 9.00) and overall acceptability (5.00 to 9.30). Jackfruit trees are cross pollinated and are mostly seed propagated. As a result, morphological and agronomical characters of jackfruit showed a distinct range of variation. The quantitative data of jackfruit germplasm and their range, mean, standard deviation (SD), standard error (SE) and coefficient of variance $(\mathrm{CV} \%)$ are presented. The highest morphological and agronomical characters was observed for trunk girth (CV 613.59\%) followed by tree height (CV $3.92 \%)$. Minimum variation was noticed in canopy spread E-W direction (CV 0.53\%), canopy spread N-S direction (CV $0.51 \%)$. The highest yield variation was observed for yield per tree (CV 22577.38\%). Total number of fruits per plant (CV 113.91\%), fruit breadth (CV 72.70\%), TSS (54.12 ${ }^{0}$ brix), fruit length (CV45.54\%). Minimum variation was noticed in individual fruit weight (CV 5.37\%). Organoleptic evaluation viz., colour and appearance (CV $1.08 \%$ ), flavor (CV 1.05\%), texture/firmness CV value (1.34\%), taste CV value $(1.57 \%)$, overall acceptability (CV 1.33\%). Among the fifty five jackfruit germplasms, three germplasm KDM-AhJ-08, KDM-AhJ-10, KDM-AhJ-46, showed good bearing and quality habit. So, the germplasm KDM-AhJ-08, KDM-AhJ-10, KDM-AhJ-46 were found to be suitable for jackfruit cultivation at dry tracts of Tamil Nadu.
\end{abstract}

\section{Keywords}

Jackfruit (J), KDM (Kudumiyanmali), Artocarpus heterophyllus (Ah)

Collection, Evaluation, germplasm, genotypes

\section{Article Info}

Accepted:

18 May 2020

Available Online:

10 June 2020

\section{Introduction}

Jackfruit (Artocarpus heterophyllus Lam.) belongs to the family Moraceae, indigenous to the rainforests of the Western Ghats of India (Rowe-Dutton, 1985). It is cultivated widely at low elevations throughout India, in many parts of Southeast Asia (Rahman et al., 1999), in the evergreen forest zone of West Africa (Burkill, 1997), and in northern Australia as well (Azad et al., 2007). In Bangladesh, it is commonly referred to as "poor man's food" (Rahman et al., 1995) as it is cheap and plentiful during the season. The nutritious seeds are boiled or roasted and eaten like chestnuts, added to flour for baking, or 
cooked in dishes. The tree is also known for its durable timber, which ages to an orange or reddish brown color, with anti-termite properties. The leaves and fruit waste provide valuable fodder for cattle, pigs and goats. Jackfruit wood chips yield a dye, which is used to give the famous orange-red color to the robes of Buddhist priests (Craig and Harley, 2006). In addition, many parts of the plant, including the bark, roots, leaves and fruits have medicinal properties (Hakim et al., 2005; Arung et al., 2006).

Despite numerous advantages, the popularity of jackfruit as a commercial crop is very poor owing to wide variations in fruit quality, the long seed dormancy and the widespread belief that excessive consumption of jackfruit flakes leads to certain digestive ailments (Samaddar, 1985). Several studies have reported diversity in jackfruit, but they were based mainly on morphological characteristics (Hossain, 1996; Saha et al., 1996, Jagadeesh et al., 2007).

Although at least 30 strains have been described in the Indian subcontinent and 30 more types in Malaysia (Singh, 1986; Vinning and Moody, 1997), only two types are recognized on the basis of fruit characteristics (Odoemelam, 2005); one has fruits with small, fibrous, soft and spongy flakes with very sweet carpels, while the other variety is crunchy, though not as sweet, with crisp carpels. This latter is considered to be of high quality, called "Koozha chakka" or "Koozha pazham" in South India.

There are no well-defined varieties in specific localities; local varieties have different names based on their variability in yield, fruit shape, flake color, total sugars, and so on. Cultivar identification and estimation of genetic diversity using morphological data are limited, as they are environmentally influenced, and there are few distinctive characters (Cavagnaro et al., 2006).
Tamil Nadu jackfruit cultivation are mainly concentrated in Cuddalore, Kanyakumari, Dindigul, Ariyalur, Thanjavur and Pudukkottai districts and they account for 73.21 per cent of area under jackfruit cultivation in Tamil Nadu. In Pudukkottai district of Tamil Nadu, Jack is planted as one of the border trees in agricultural and horticultural crop lands. It is one of the most drought tolerant, hardy fruit crop and traditionally it is a farmer's house hold fruit. Owing to it are various culinary uses and availability in plenty during the summer season.

The young fruit has great demand as vegetable whereas the ripe fruit is a good source of vitamins and minerals. Jack fruit is also gaining popularity in Mumbai market extends sweet welcome to (Vadakadu, Mangadu, Periyavadi, Vanakkankadu, Vandanviduthy, Pullanviduthy, Senthangudi, Kothamangalam, Keeramngalam, Panankulam, Maramadakki, Anavayal, Neduvasal and Karukakurichi of Pudukkottai district) In Pudukkottai district of Tamil Nadu due to emerging ethnic and mainstream marketing opportunities (The Hindu., 2015).

In Pudukkottai district the area under Jackfruit is 1102 ha (Department of Statistical, Pudukkottai, 2018) with the productivity of 7.00 tonnes/ ha. The farmers are growing the local varieties without knowing its productivity. Sometimes unknowingly the farmers choose the poor yielding seedlings and unable to get good yield.

To overcome this, there is an urgent need to identify a best variety suitable for this region to improve the income of the jack farmers. Hence, Owing to its uses and ease of cultivation in dry districts of Tamil Nadu, identification of a variety with high yield and superior quality is the need of the hour. 
With this background, the research on Identification of regular bearing, high yielding jackfruit genotypes with superior fruit quality is formulated. A study was therefore, carried out to find out a suitable jackfruit genotypes for further variety development programme at Pudukkottai District in Tamil Nadu.

\section{Materials and Methods}

Fifty five jackfruit germplasm viz., KDMAhJ-01 to KDM-AhJ-55 were selected for the study to identify superior ones. The recorded age of the selected tress ranged from 07 to 33 years. Morphological characters viz., age of the plant, tree height $(\mathrm{m})$, trunk girth $(\mathrm{cm})$, canopy spread E- W direction (m), canopy spread N-S direction (m), month of flowering, month of harvest. Yield and quality quantitative characters viz., number of fruits per tree, individual fruit weight $(\mathrm{kg})$, yield per tree $(\mathrm{kg})$, fruit length $(\mathrm{cm})$, fruit breadth $(\mathrm{cm})$ and TSS ( ${ }^{0}$ brix), colour and appearance, flavor, texture/firmness, taste, overall acceptability were recorded and statistically analyzed. The compiled data were analyzed for measuring range, mean, standard deviation (SD), standard error of mean (SE) and coefficient of variance (CV \%) using Microsoft Office Excel package.

\section{Results and Discussion}

According to the International Plant Genetic Resources Institute [IPGRI, 2000] morphological variation among jackfruit trees was recorded using jackfruit descriptor. Growth, yield and qualitative measures of vegetative and reproductive characters of jackfruit were measured in the descriptors.

The growth and yield characters were measured by observation. The qualitative fruit characters were measured by eye observation and organoleptic test.

\section{Growth characters}

Growth, yield and qualitative were recorded and evaluated to know the genetic variability among the studied fifty five jackfruit germplasms. The jackfruit germplasm evaluated in the present study showed the variability for all the growth characters traits studied. Wide range of variation was observed among the germplasm in terms of age of the plant, tree height $(\mathrm{m})$, trunk girth $(\mathrm{cm})$, canopy spread E-W direction (m), canopy spread N-S direction (m), month of flowering and month of harvest (Table 1). Yield and quality characters viz, numbers of fruits per tree, individual fruit weight, yield per tree, fruit length, fruit breadth and TSS were presented (Table 2). Quality characters viz., colour and appearance, flavor, texture/firmness, taste, overall acceptability was presented (Table 3).

The age of plant of fifty five jackfruit genotypes was greatly significantly varied. Age of tree ranged from 07.00 to 33.00 years. The germplasm KDM-AhJ-31 and KDMAhJ-32 was the oldest aged plant (33.00 years), while KDM-AhJ -16, KDM-AhJ-17, KDM-AhJ-18, and KDM-AhJ-19 germplasm was the 7.00 years old plant. The yield of jackfruit tree increases with the increase of age of the plant.Tree height of the fifty five jackfruit genotypes was greatly significantly varied. Tree height varied from $2.20 \mathrm{~m}$ to $10.30 \mathrm{~m}$. The germplasm KDM-AhJ-19 was the lowest plant height $2.20 \mathrm{~m}$, while the genotype KDM-AhJ-52 recorded the highest tree height $10.25 \mathrm{~m}$. Trunk girth was differing significantly. The highest trunk girth was observed in KDM-AhJ-38 $(158.00 \mathrm{~cm})$. The genotype KDM-AhJ-04 was recorded for the lowest trunk girth of $55.00 \mathrm{~cm}$. The yield of jackfruit tree increases with the increase of tree trunk girth of the plant (Table 1). Higher stem circumference gives better support for the main branches and reflects the vigour of 
trees indirectly, bearing more number of fruits lead to higher yield. Lesser the trunk height with spreading nature, greater will be the number of fruits per tree due to more primary and secondary branches. Similar variability was reported by Muthulakshmi (2003), Murugan (2007) in jackfruit.

A diversity of canopy spread was observed among the genotypes. Significance variation was observed in case of canopy spread E-W direction of $1.20 \mathrm{~m}$ to $3.60 \mathrm{~m}$. The genotypes KDM-AhJ-36 and KDM-AhJ-46 was observed the highest canopy spread of $3.60 \mathrm{~m}$ and the lowest of $1.15 \mathrm{~m}$ of KDM-AhJ-30. The yield of jackfruit plant increases with the increase of canopy spread of the plant. The highest canopy spread of $3.60 \mathrm{~m}$ was observed by the germplasm KDM-AhJ-01 and the lowest of $1.12 \mathrm{~m}$ by KDM-AhJ-30. The yield of jackfruit plant increases due to the highest canopy spread for the East-West and North -South direction. This may be due to genetic nature of the genotypes and growing environment.

Based on the fruiting season, the selected genotypes were grouped into early season (January-March), mid-season (April -June) and late season (July-August). Among the genotypes, majority of the genotypes showed early season bearing habit. Whereas KDMAhJ-46 mid-season bearer. Meanwhile, KDM-AhJ-10 flowering was started by the month of January -February and harvest was extended to the month August to September. The genotype KDM-AhJ-10 was late season bearer (Table 1).

\section{Yield characters}

Highly significant variation was observed among the genotypes in terms of fruit characters. Highest number of fruits per plant (50.00) was obtained from KDM-AhJ-10 followed by KDM-AhJ-46 (45.00) and the minimum of (10.00) was from KDM-AhJ-16. Yield is directly correlated with the number of fruits and weight of the individual fruit (Table 2).Individual fruit weight was differing significantly. Maximum individual fruit weight was gained from KDM-AhJ- 46 (18.00 $\mathrm{kg})$ followed by KDM-AhJ-10 (16.00 kg) and minimum of $(7.00 \mathrm{~kg})$ was observe by the genotype KDM-AhJ-08. Nowadays small sized jackfruit is preferred due to small family size. Farmer's point of view medium and large sized fruits are economically viable and export market is more preferable (Table 2).

Fruit length and breadth of fifty five jackfruit were significantly varied (Table 2). Fruit length ranged from 11.00 to $45.00 \mathrm{~cm}$ and breadth 18.00 to $57.00 \mathrm{~cm}$ with the mean of $24.36 \mathrm{~cm}$ and $33.73 \mathrm{~cm}$, respectively. The longest fruit was obtained from KDM-AhJ-46 $(45.00 \mathrm{~cm})$ followed by the KDM-AhJ-10 $(40.00 \mathrm{~cm})$ and shortest fruit in KDM-AhJ-25 $(12.00 \mathrm{~cm})$. The germplasm KDM-AhJ-46 produced the wider fruit breadth $(57.00 \mathrm{~cm})$ followed by KDM-AhJ-10 $(50.00 \mathrm{~cm})$ and narrower fruit in KDM-AhJ $-06(18.00 \mathrm{~cm})$. TSS ranged from 6.00 to 29.00 with mean value of 14.36 (Table 2). Maximum TSS (29 ${ }^{0}$ brix) was obtained from KDM-AhJ -10 and KDM-AhJ -46 and minimum (06 ${ }^{\circ}$ brix) was found from the germplasm KDM-AhJ-51.

\section{Quality characters}

Significant variation was noticed among the fifty five jackfruit germplasms in terms of weight of rind (Table 3). Weight of rind ranged from 3.00 to $8.00 \mathrm{~kg}$ with an average value of $5.80 \mathrm{~kg}$. The highest weight of rind was found from KDM-Ah9 and KDM-Ah11 $(8.00 \mathrm{~kg})$ and lowest in KDM-Ah04 and KDM-Ah08 $(3.00 \mathrm{~kg})$. Weight of rind is directly proportionate to edible portion of the fruit of jackfruit germplasm. Significant variation was found in case of number of flakes per fruit. 
It ranged from 30.00 to 210.00 with mean value of 81.00 (Table 3). Maximum number of flakes per fruit was obtained from KDMAh 46 (210.00) followed by the germplasm KDM-Ah10 (150.00) and minimum was from AHJ-32, KDM-Ah33 (40.00).

Total flakes weight per fruit contrasted expressively among the fifty five jackfruit germplasms. Maximum total flakes weight per fruit was originated in KDM-Ah 46 $(13.60 \mathrm{~kg})$ and the minimum in KDM-Ah 17 $(3.10 \mathrm{~kg})$. Significant variation was found in case of weight of individual flake with seed.

Individual flake with seed ranged from 20.00 $\mathrm{gm}$ to $170.00 \mathrm{gm}$ with mean value of 71.29.gm. Maximum weight of individual flake with seed was obtained from KDM-Ah 49 (110.00 gm) and minimum of $20.00 \mathrm{gm}$ was found from the germplasm KDM-Ah07.

Individual seed weight ranged from $08.00 \mathrm{gm}$ to $60.00 \mathrm{gm}$ with mean value of $25.24 \mathrm{~g}$. Maximum weight of individual seed weight was obtained from KDM-Ah -32 (65.00 gm) and minimum of $09.00 \mathrm{gm}$ was found from the germplasm KDM-Ah-06.

Flakes: seed ranged from 0.42 .00 to 7.00 with mean value of 2.28. Maximum Flakes: seed ratio was obtained from KDM-Ah -10 (5.30) and minimum of 0.42 was found from the germplasm KDM-Ah12. TSS ranged from 6.00 to 29.00 with mean value of 14.36 . Maximum TSS ( $2^{0}$ brix) was obtained from KDM-Ah -10 and KDM-Ah -46 and minimum of $\left(6^{0}\right.$ brix $)$ was found from the germplasm KDM-Ah-51.Colour and appearance ranged from 4.00 to 9.00 with mean value of 6.40. Maximum colour and appearance 9.00 was obtained from KDMAhJ -10 and minimum 4.00 was found from the germplasm KDM-AhJ-43. Flavor ranged from 4.00 to 9.00 with mean value of 6.66. Maximum flavor 9.00 was obtained from
KDM-AhJ -10 and minimum 4.00 was found from the genotypes KDM-AhJ-36 and KDMAhJ-48. Texture/Firmness ranged from 4.00 to 9.00 with mean value of 6.31. Maximum texture /firmness 9.00 were obtained from KDM-AhJ -10 and the minimum 4.00 was recorded by the genotypes KDM-Ah-37 and KDM-Ah-38.

Taste ranged from 3.00 to 9.00 with mean value of 6.21. Maximum taste 9.00 was obtained from KDM-Ah -10 followed by KDM-Ah -46 and minimum of 3.00 was found from the germplasm KDM-Ah-37. Overall acceptability ranged from 5.00 to 9.30 with mean value of 6.31. Maximum overall acceptability 9.30 was observed by the genotype KDM-AhJ -10 followed by KDMAh J-46 (9.20) and minimum 5.00 was observed by the germplasm KDM-AhJ-36 and KDM-AhJ-51.

The organoleptic test is the final judgment for acceptance of the fruit quality of the selected genotypes. The present study revealed that remarkable variation by organoleptic evaluation in all the characters of flakes. Genotypes namely KDM-AhJ-10 (9.30) followed by KDM-AhJ-46 (9.20) have higher score for overall quality of flakes. This may be due to genetic nature of the genotypes and growing environment. Similar findings were reported by Murugan (2007). Fruit shape varied from oval, oval, round and slender. The genotypes KDM-Ah -10 and KDM-Ah 46 record the slender shape of fruits. (Table $5)$.

Flakes colour varied distinctly among the fifty five jackfruit germplasms. Flakes colour varied from yellow, whitish yellow, light yellow, bright yellow. The genotype KDMAh -10 is bright yellow/Senthuram. Shelf life of flakes varied distinctly among the fifty five jackfruit germplasms. The genotypes KDMAh -10 and KDM-Ah -46 record for five days. 
Table.1 Growth characters of jackfruit genotypes KDM-Ah-1 to KDM-Ah-55

\begin{tabular}{|c|c|c|c|c|c|c|c|}
\hline $\begin{array}{l}\text { Acc. } \\
\text { No. }\end{array}$ & $\begin{array}{l}\text { Age of the } \\
\text { tree }\end{array}$ & $\begin{array}{c}\text { Tree } \\
\text { height (m) }\end{array}$ & $\begin{array}{c}\text { Trunk } \\
\text { girth }(\mathrm{cm})\end{array}$ & $\begin{array}{c}\text { Canopy } \\
\text { spread } \\
\text { E-W (m) }\end{array}$ & $\begin{array}{c}\text { Canopy } \\
\text { spread N- } \\
\text { S (m) }\end{array}$ & $\begin{array}{l}\text { Month } \\
\text { of flowering }\end{array}$ & $\begin{array}{l}\text { Month of } \\
\text { harvest }\end{array}$ \\
\hline KDM-AhJ-1 & 25.00 & 9.00 & 110.00 & 3.50 & 3.60 & $\begin{array}{l}\text { November- } \\
\text { December }\end{array}$ & March-June \\
\hline KDM-AhJ-2 & 20.00 & 5.60 & 105.00 & 2.50 & 2.90 & $\begin{array}{l}\text { November } \\
\text {-December }\end{array}$ & March-June \\
\hline KDM-AhJ-3 & 25.00 & 5.70 & 110.00 & 3.60 & 2.90 & $\begin{array}{l}\text { November } \\
\text {-December }\end{array}$ & March-June \\
\hline KDM-AhJ-4 & 22.00 & 3.60 & 55.00 & 2.60 & 2.70 & $\begin{array}{l}\text { November } \\
\text {-December }\end{array}$ & March-June \\
\hline KDM-AhJ-5 & 24.00 & 7.90 & 100.00 & 3.50 & 2.40 & $\begin{array}{l}\text { November } \\
\text {-December }\end{array}$ & March-June \\
\hline KDM-AhJ-6 & 20.00 & 5.60 & 65.00 & 1.90 & 2.50 & $\begin{array}{l}\text { November } \\
\text {-December }\end{array}$ & March-June \\
\hline KDM-AhJ-7 & 20.00 & 6.60 & 70.00 & 1.60 & 1.80 & $\begin{array}{l}\text { November } \\
\text {-December }\end{array}$ & March-June \\
\hline KDM-AhJ-8 & 27.00 & 8.10 & 75.00 & 3.00 & 3.20 & $\begin{array}{l}\text { November } \\
\text {-December }\end{array}$ & March-June \\
\hline KDM-Ah-9 & 30.00 & 8.50 & 76.00 & 2.70 & 2.90 & $\begin{array}{l}\text { November } \\
\text {-December }\end{array}$ & March-June \\
\hline KDM-AhJ-10 & 30.00 & 7.50 & 76.00 & 3.40 & 3.50 & $\begin{array}{l}\text { January } \\
\text { February }\end{array}$ & July-September \\
\hline KDM-AhJ-11 & 30.00 & 6.50 & 68.00 & 1.90 & 2.50 & $\begin{array}{l}\text { November } \\
\text {-December }\end{array}$ & March-June \\
\hline KDM-AhJ-12 & 26.00 & 7.50 & 70.00 & 1.40 & 1.90 & $\begin{array}{l}\text { November } \\
\text {-December }\end{array}$ & March-June \\
\hline KDM-AhJ-13 & 25.00 & 5.40 & 72.00 & 2.20 & 2.40 & $\begin{array}{l}\text { November } \\
\text {-December }\end{array}$ & March-June \\
\hline KDM-AhJ-14 & 20.00 & 5.20 & 70.00 & 1.70 & 1.72 & $\begin{array}{l}\text { November } \\
\text {-December }\end{array}$ & March-June \\
\hline KDM-AhJ-15 & 24.00 & 5.42 & 72.00 & 1.50 & 1.60 & $\begin{array}{l}\text { November } \\
\text {-December }\end{array}$ & March-June \\
\hline KDM-AhJ-16 & 7.00 & 3.40 & 45.00 & 1.50 & 1.40 & $\begin{array}{l}\text { November } \\
\text {-December }\end{array}$ & March-June \\
\hline KDM-AhJ-17 & 7.00 & 5.40 & 105.00 & 2.50 & 3.50 & $\begin{array}{l}\text { November } \\
\text {-December }\end{array}$ & March-June \\
\hline KDM-AhJ-18 & 7.00 & 3.40 & 75.00 & 1.80 & 1.42 & $\begin{array}{l}\text { November } \\
\text {-December }\end{array}$ & March-June \\
\hline KDM-AhJ-19 & 7.00 & 2.20 & 72.00 & 1.40 & 1.50 & $\begin{array}{l}\text { November - } \\
\text { December }\end{array}$ & March-June \\
\hline KDM-AhJ-20 & 10.00 & 4.40 & 105.00 & 2.20 & 1.72 & $\begin{array}{l}\text { November } \\
\text {-December }\end{array}$ & March-June \\
\hline KDM-AhJ-21 & 10.00 & 6.40 & 110.00 & 1.72 & 1.80 & $\begin{array}{l}\text { November } \\
\text {-December }\end{array}$ & March-June \\
\hline KDM-AhJ-22 & 20.00 & 5.30 & 95.00 & 1.30 & 1.42 & $\begin{array}{l}\text { November } \\
\text {-December }\end{array}$ & March-June \\
\hline
\end{tabular}




\begin{tabular}{|c|c|c|c|c|c|c|c|}
\hline KDM-AhJ-23 & 20.00 & 5.50 & 70.00 & 1.50 & 1.60 & $\begin{array}{l}\text { November } \\
\text {-December }\end{array}$ & March-June \\
\hline KDM-AhJ-24 & 10.00 & 4.15 & 105.00 & 1.40 & 1.65 & $\begin{array}{l}\text { November } \\
\text {-December }\end{array}$ & March-June \\
\hline KDM-AhJ-25 & 15.00 & 5.25 & 100.00 & 1.20 & 1.30 & $\begin{array}{l}\text { November } \\
\text {-December }\end{array}$ & March-June \\
\hline KDM-AhJ-26 & 15.00 & 4.40 & 80.00 & 1.40 & 1.45 & $\begin{array}{l}\text { November } \\
\text {-December }\end{array}$ & March-June \\
\hline KDM-AhJ-27 & 15.00 & 4.32 & 75.00 & 1.42 & 1.65 & $\begin{array}{l}\text { November } \\
\text {-December }\end{array}$ & March-June \\
\hline KDM-AhJ-28 & 15.00 & 3.20 & 70.00 & 1.20 & 1.25 & $\begin{array}{l}\text { November } \\
\text {-December }\end{array}$ & March-June \\
\hline KDM-AhJ-29 & 12.00 & 3.40 & 58.00 & 1.30 & 1.70 & $\begin{array}{l}\text { November } \\
\text {-December }\end{array}$ & March-June \\
\hline KDM-AhJ-30 & 12.00 & 8.30 & 100.00 & 1.15 & 1.12 & $\begin{array}{l}\text { November } \\
\text {-December }\end{array}$ & March-June \\
\hline KDM-AhJ-31 & 33.00 & 9.20 & 110.00 & 2.90 & 1.90 & $\begin{array}{l}\text { November } \\
\text {-December }\end{array}$ & March-June \\
\hline KDM-AhJ-32 & 33.00 & 6.25 & 97.00 & 3.12 & 3.15 & $\begin{array}{l}\text { November } \\
\text {-December }\end{array}$ & March-June \\
\hline KDM-AhJ-33 & 26.00 & 4.30 & 110.00 & 1.40 & 1.30 & $\begin{array}{l}\text { November } \\
\text {-December }\end{array}$ & March-June \\
\hline KDM-AhJ-34 & 25.00 & 7.20 & 56.00 & 1.20 & 1.40 & $\begin{array}{l}\text { November } \\
\text {-December }\end{array}$ & March-June \\
\hline KDM-AhJ-35 & 25.00 & 5.20 & 70.00 & 2.20 & 2.65 & $\begin{array}{l}\text { November } \\
\text {-December }\end{array}$ & March-June \\
\hline KDM-AhJ-36 & 25.00 & 6.10 & 122.00 & 3.60 & 3.80 & $\begin{array}{l}\text { November } \\
\text {-December }\end{array}$ & March-June \\
\hline KDM-AhJ-37 & 25.00 & 5.10 & 145.00 & 2.25 & 2.20 & $\begin{array}{l}\text { November } \\
\text {-December }\end{array}$ & March-June \\
\hline KDM-AhJ-38 & 20.00 & 8.10 & 158.00 & 2.60 & 2.75 & $\begin{array}{l}\text { November } \\
\text {-December }\end{array}$ & March-June \\
\hline KDM-AhJ-39 & 15.00 & 10.20 & 135.00 & 2.15 & 2.30 & $\begin{array}{l}\text { November } \\
\text {-December }\end{array}$ & March-June \\
\hline KDM-AhJ-40 & 12.00 & 7.20 & 147.00 & 3.30 & 3.20 & $\begin{array}{l}\text { November } \\
\text {-December }\end{array}$ & March-June \\
\hline KDM-AhJ-41 & 20.00 & 5.20 & 65.00 & 1.65 & 1.85 & $\begin{array}{l}\text { November } \\
\text {-December }\end{array}$ & March-June \\
\hline KDM-AhJ-42 & 15.00 & 10.20 & 128.00 & 2.32 & 2.25 & $\begin{array}{l}\text { November } \\
\text {-December }\end{array}$ & March-June \\
\hline KDM-AhJ-43 & 20.00 & 5.15 & 95.00 & 2.30 & 2.10 & $\begin{array}{l}\text { November } \\
\text {-December }\end{array}$ & March-June \\
\hline KDM-AhJ-44 & 20.00 & 8.50 & 110.00 & 3.35 & 3.50 & $\begin{array}{l}\text { November } \\
\text {-December }\end{array}$ & March-June \\
\hline KDM-AhJ-45 & 15.00 & 7.20 & 75.00 & 2.30 & 2.40 & $\begin{array}{l}\text { November } \\
\text {-December }\end{array}$ & March-June \\
\hline KDM-AhJ-46 & 30.00 & 7.60 & 100.00 & 3.60 & 3.50 & $\begin{array}{l}\text { November } \\
\text {-December }\end{array}$ & April-June \\
\hline KDM-AhJ-47 & 20.00 & 7.20 & 80.00 & 1.70 & 1.85 & November & March-June \\
\hline
\end{tabular}




\begin{tabular}{|c|c|c|c|c|c|c|c|}
\hline & & & & & & -December & \\
\hline KDM-AhJ-48 & 22.00 & 9.10 & 80.00 & 2.20 & 2.30 & $\begin{array}{l}\text { November } \\
\text {-December }\end{array}$ & March-June \\
\hline KDM-AhJ-49 & 22.00 & 8.20 & 80.00 & 1.90 & 2.20 & $\begin{array}{l}\text { November } \\
\text {-December }\end{array}$ & March-June \\
\hline KDM-AhJ-50 & 25.00 & 10.20 & 65.00 & 1.40 & 1.30 & $\begin{array}{l}\text { November } \\
\text {-December }\end{array}$ & March-June \\
\hline KDM-AhJ-51 & 25.00 & 8.30 & 90.10 & 2.20 & 2.30 & $\begin{array}{l}\text { November } \\
\text {-December }\end{array}$ & March-June \\
\hline KDM-AhJ-52 & 30.00 & 10.25 & 120.00 & 2.30 & 2.40 & $\begin{array}{l}\text { November } \\
\text {-December }\end{array}$ & March-June \\
\hline KDM-AhJ-53 & 20.00 & 5.30 & 95.00 & 1.90 & 1.75 & $\begin{array}{l}\text { November } \\
\text {-December }\end{array}$ & March-June \\
\hline KDM-AhJ-54 & 27.00 & 6.20 & 115.00 & 2.30 & 2.20 & $\begin{array}{l}\text { November } \\
\text {-December }\end{array}$ & March-June \\
\hline KDM-AhJ- 55 & 25.00 & 7.30 & 105.00 & 2.20 & 1.70 & $\begin{array}{l}\text { November } \\
\text {-December }\end{array}$ & March-June \\
\hline Max. & 33.00 & 10.30 & 158.00 & 3.60 & 3.80 & - & - \\
\hline Min. & 7.00 & 2.20 & 45.00 & 1.20 & 1.12 & - & - \\
\hline Mean & 20.50 & 6.40 & 91.13 & 2.20 & 2.20 & - & - \\
\hline SD & 6.9 & 2.00 & 24.99 & 0.73 & 0.72 & - & - \\
\hline SE $( \pm)$ & 3.43 & 0.49 & 0.86 & 0.34 & 0.37 & - & - \\
\hline CV (\%) & 47.88 & 3.92 & 613.59 & 0.53 & 0.51 & - & - \\
\hline
\end{tabular}

Table.2 Yield and quality characters of jackfruit genotypes KDM-Ah-1 to KDM-Ah-55

\begin{tabular}{|c|c|c|c|c|c|c|}
\hline $\begin{array}{l}\text { Acc. } \\
\text { No. }\end{array}$ & $\begin{array}{c}\text { No.of } \\
\text { fruits } \\
\text { /tree }\end{array}$ & $\begin{array}{l}\text { Individual } \\
\text { fruit } \\
\text { weight (kg) }\end{array}$ & $\begin{array}{c}\text { Yield } \\
\text { /tree (kg) }\end{array}$ & $\begin{array}{c}\text { Fruit } \\
\text { Length } \\
\text { (cm) }\end{array}$ & $\begin{array}{l}\text { Fruit } \\
\text { breadth } \\
\text { (cm) }\end{array}$ & $\begin{array}{c}\text { TSS } \\
\left({ }^{0} \text { brix }\right)\end{array}$ \\
\hline KDM-Ah-1 & 12.00 & 10.00 & 120.00 & 30.00 & 42.00 & 10.00 \\
\hline KDM-Ah-2 & 18.00 & 11.00 & 198.00 & 25.00 & 36.00 & 11.00 \\
\hline KDM-Ah-3 & 15.00 & 12.00 & 180.00 & 27.00 & 45.00 & 10.00 \\
\hline KDM-Ah-4 & 20.00 & 8.00 & 160.00 & 16.00 & 25.00 & 20.00 \\
\hline KDM-Ah-5 & 14.00 & 12.00 & 168.00 & 24.00 & 47.00 & 9.00 \\
\hline KDM-Ah-6 & 14.00 & 8.00 & 112.00 & 14.00 & 18.00 & 7.00 \\
\hline KDM-Ah-7 & 13.00 & 9.00 & 117.00 & 18.00 & 30.00 & 24.00 \\
\hline KDM-Ah-8 & 50.00 & 7.00 & 350.00 & 26.00 & 32.00 & 26.00 \\
\hline KDM-Ah-9 & 18.00 & 15.00 & 270.00 & 30.00 & 23.00 & 12.00 \\
\hline KDM-Ah-10 & 40.00 & 16.00 & 640.00 & 40.00 & 50.00 & 29.00 \\
\hline KDM-Ah-11 & 32.00 & 15.00 & 480.00 & 14.00 & 24.00 & 10.00 \\
\hline KDM-Ah-12 & 30.00 & 12.00 & 360.00 & 22.00 & 34.00 & 10.00 \\
\hline KDM-Ah-13 & 28.00 & 11.00 & 308.00 & 22.00 & 31.00 & 22.00 \\
\hline KDM-Ah-14 & 30.00 & 12.00 & 360.00 & 22.00 & 34.00 & 12.00 \\
\hline KDM-Ah-15 & 21.00 & 9.00 & 189.00 & 26.00 & 31.00 & 13.00 \\
\hline KDM-Ah-16 & 10.00 & 8.00 & 80.00 & 25.00 & 32.00 & 8.00 \\
\hline KDM-Ah-17 & 14.00 & 8.00 & 112.00 & 32.00 & 24.00 & 10.00 \\
\hline KDM-Ah-18 & 11.00 & 9.00 & 99.00 & 26.00 & 31.00 & 7.00 \\
\hline
\end{tabular}




\begin{tabular}{|c|c|c|c|c|c|c|}
\hline KDM-Ah-19 & 15.00 & 8.00 & 120.00 & 23.00 & 28.00 & 9.00 \\
\hline KDM-Ah-20 & 16.00 & 9.00 & 144.00 & 26.00 & 31.00 & 7.00 \\
\hline KDM-Ah-21 & 13.00 & 8.00 & 104.00 & 21.00 & 44.00 & 7.00 \\
\hline KDM-Ah-22 & 13.00 & 10.00 & 130.00 & 23.00 & 30.00 & 7.00 \\
\hline KDM-Ah-23 & 15.00 & 10.00 & 150.00 & 28.00 & 40.00 & 8.00 \\
\hline KDM-Ah-24 & 12.00 & 9.00 & 108.00 & 21.00 & 28.00 & 7.00 \\
\hline KDM-Ah-25 & 19.00 & 9.00 & 171.00 & 12.00 & 25.00 & 7.00 \\
\hline KDM-Ah-26 & 30.00 & 8.00 & 240.00 & 24.00 & 32.00 & 7.00 \\
\hline KDM-Ah-27 & 33.00 & 13.00 & 429.00 & 26.00 & 34.00 & 18.00 \\
\hline KDM-Ah-28 & 34.00 & 11.00 & 374.00 & 26.00 & 33.00 & 12.00 \\
\hline KDM-Ah-29 & 28.00 & 12.00 & 336.00 & 33.00 & 40.00 & 24.00 \\
\hline KDM-Ah-30 & 30.00 & 10.00 & 300.00 & 28.00 & 50.00 & 18.00 \\
\hline KDM-Ah31 & 42.00 & 8.00 & 336.00 & 11.00 & 44.00 & 27.00 \\
\hline KDM-Ah-32 & 25.00 & 11.00 & 275.00 & 22.00 & 27.00 & 23.00 \\
\hline KDM-Ah-33 & 32.00 & 8.00 & 256.00 & 12.00 & 27.00 & 12.00 \\
\hline KDM-Ah-34 & 38.00 & 9.00 & 342.00 & 33.00 & 42.00 & 21.00 \\
\hline KDM-Ah-35 & 34.00 & 12.00 & 408.00 & 30.00 & 47.00 & 9.00 \\
\hline KDM-Ah-36 & 18.00 & 10.00 & 180.00 & 32.00 & 47.00 & 10.00 \\
\hline KDM-Ah-37 & 14.00 & 8.00 & 112.00 & 27.00 & 36.00 & 7.00 \\
\hline KDM-Ah-38 & 12.00 & 10.00 & 120.00 & 17.00 & 26.00 & 20.00 \\
\hline KDM-Ah-39 & 13.00 & 12.00 & 156.00 & 20.00 & 30.00 & 10.00 \\
\hline KDM-Ah-40 & 17.00 & 8.00 & 136.00 & 22.00 & 26.00 & 20.00 \\
\hline KDM-Ah-41 & 19.00 & 9.00 & 171.00 & 30.00 & 42.00 & 26.00 \\
\hline KDM-Ah-42 & 40.00 & 10.00 & 400.00 & 18.00 & 30.00 & 24.00 \\
\hline KDM-Ah-43 & 22.00 & 9.00 & 198.00 & 30.00 & 45.00 & 9.00 \\
\hline KDM-Ah-44 & 40.00 & 13.00 & 520 & 32.00 & 34.00 & 28.00 \\
\hline KDM-Ah-45 & 32.00 & 11.00 & 352.00 & 27.00 & 38.00 & 20.00 \\
\hline KDM-Ah-46 & 45.00 & 18.00 & 810.00 & 45.00 & 57.00 & 29.00 \\
\hline KDM-Ah-47 & 18.00 & 14.00 & 252.00 & 25.00 & 32.00 & 17.00 \\
\hline KDM-Ah-48 & 22.00 & 10.00 & 220.00 & 32.00 & 36.00 & 10.00 \\
\hline KDM-Ah-49 & 24.00 & 14.00 & 336.00 & 27.00 & 34.00 & 10.00 \\
\hline KDM-Ah-50 & 22.00 & 12.00 & 264.00 & 17.00 & 23.00 & 7.00 \\
\hline KDM-Ah-51 & 20.00 & 9.00 & 180.00 & 19.00 & 23.00 & 6.00 \\
\hline KDM-Ah-52 & 38.00 & 12.00 & 456.00 & 17.00 & 26.00 & 7.00 \\
\hline KDM-Ah-53 & 32.00 & 9.00 & 288.00 & 15.00 & 20.00 & 8.00 \\
\hline KDM-Ah-54 & 43.00 & 12.00 & 516.00 & 22.00 & 26.00 & 25.00 \\
\hline KDM-Ah- 55 & 42.00 & 11.00 & 462.00 & 28.00 & 33.00 & 24.00 \\
\hline Max. & 50.00 & 18.00 & 810.00 & 45.00 & 57.00 & 29.00 \\
\hline Min. & 10.00 & 7.00 & 80.00 & 11.00 & 18.00 & 6.00 \\
\hline Mean & 24.58 & 10.51 & 266.45 & 24.36 & 33.73 & 14.36 \\
\hline SD & 10.77 & 2.34 & 151.64 & 6.81 & 8.60 & 7.42 \\
\hline $\mathrm{SE}( \pm)$ & 0.80 & 0.59 & 3.44 & 0.81 & 0.62 & 0.67 \\
\hline CV (\%) & 113.91 & 5.37 & 22577.38 & 45.54 & 72.71 & 54.12 \\
\hline
\end{tabular}


Table.3 Yield and quality characters of jackfruit genotypes KDM-Ah-1 to KDM-Ah-55

\begin{tabular}{|c|c|c|c|c|c|c|c|}
\hline $\begin{array}{l}\text { Acc. } \\
\text { No. }\end{array}$ & $\begin{array}{l}\text { Fruit } \\
\text { rind } \\
\text { weight } \\
\text { (kg) }\end{array}$ & $\begin{array}{l}\text { Number } \\
\text { of flakes } \\
\text { per fruit }\end{array}$ & $\begin{array}{l}\text { Total } \\
\text { weight of } \\
\text { flakes per } \\
\text { fruit }(\mathbf{k g})\end{array}$ & $\begin{array}{l}\text { Weight of } \\
\text { individual } \\
\text { flake with } \\
\text { seed (g) }\end{array}$ & $\begin{array}{l}\text { Weight of } \\
\text { individual } \\
\text { flake } \\
\text { without } \\
\text { seed (g) }\end{array}$ & $\begin{array}{l}\text { Seed } \\
\text { weight } \\
\text { (g) }\end{array}$ & $\begin{array}{l}\text { Flakes: } \\
\text { seed ratio }\end{array}$ \\
\hline KDM-AhJ-1 & 4.50 & 100.00 & 5.50 & 55.00 & 40.00 & 15.00 & 2.66 \\
\hline KDM-AhJ-2 & 5.00 & 75.00 & 6.00 & 80.00 & 55.00 & 25.00 & 2.20 \\
\hline KDM-AhJ-3 & 6.50 & 120.00 & 5.50 & 45.00 & 30.00 & 15.00 & 2.00 \\
\hline KDM-AhJ-4 & 3.00 & 100.00 & 5.00 & 50.00 & 40.00 & 10.00 & 4.00 \\
\hline KDM-AhJ-5 & 7.00 & 75.00 & 5.00 & 66.00 & 50.00 & 16.00 & 3.10 \\
\hline KDM-AhJ-6 & 4.50 & 105.00 & 3.50 & 33.00 & 25.00 & 9.00 & 2.70 \\
\hline KDM-AhJ-7 & 5.50 & 100.00 & 3.50 & 35.00 & 20.00 & 15.00 & 1.30 \\
\hline KDM-AhJ-8 & 3.00 & 75.00 & 5.00 & 53.00 & 43.00 & 10.00 & 4.30 \\
\hline KDM-Ah-9 & 8.00 & 101.00 & 7.00 & 69.00 & 40.00 & 29.00 & 1.40 \\
\hline KDM-AhJ-10 & 5.00 & 150.00 & 11.00 & 83.00 & 70.00 & 13.00 & 5.30 \\
\hline KDM-AhJ-11 & 8.00 & 100.00 & 7.00 & 70.00 & 45.00 & 25.00 & 1.80 \\
\hline KDM-AhJ-12 & 6.50 & 130.00 & 6.50 & 50.00 & 15.00 & 35.00 & 0.42 \\
\hline KDM-AhJ-13 & 5.50 & 80.00 & 5.50 & 69.00 & 50.00 & 19.00 & 2.60 \\
\hline KDM-AhJ-14 & 7.00 & 100.00 & 5.00 & 50.00 & 35.00 & 15.00 & 2.30 \\
\hline KDM-AhJ-15 & 5.00 & 120.00 & 4.00 & 33.00 & 20.00 & 13.00 & 1.50 \\
\hline KDM-AhJ-16 & 4.50 & 65.00 & 3.50 & 54.00 & 40.00 & 14.00 & 2.80 \\
\hline KDM-AhJ-17 & 5.00 & 50.00 & 3.10 & 60.00 & 45.00 & 15.00 & 3.00 \\
\hline KDM-AhJ-18 & 4.50 & 50.00 & 4.50 & 90.00 & 50.00 & 40.00 & 1.25 \\
\hline KDM-AhJ-19 & 5.00 & 60.00 & 3.10 & 50.00 & 35.00 & 15.00 & 2.30 \\
\hline KDM-AhJ-20 & 5.00 & 50.00 & 4.00 & 80.00 & 55.00 & 25.00 & 2.20 \\
\hline KDM-AhJ-21 & 5.00 & 105.00 & 3.00 & 28.00 & 18.00 & 10.00 & 1.80 \\
\hline KDM-AhJ-22 & 6.00 & 55.00 & 4.00 & 72.00 & 40.00 & 32.00 & 1.25 \\
\hline KDM-AhJ-23 & 5.50 & 45.00 & 5.50 & 122.00 & 62.00 & 60.00 & 1.03 \\
\hline KDM-AhJ-24 & 5.00 & 45.00 & 4.00 & 88.00 & 40.00 & 48.00 & 0.83 \\
\hline KDM-AhJ-25 & 6.00 & 30.00 & 3.10 & 100.00 & 55.00 & 45.00 & 1.22 \\
\hline KDM-AhJ-26 & 5.00 & 50.00 & 3.30 & 60.00 & 35.00 & 25.00 & 1.40 \\
\hline KDM-AhJ-27 & 7.00 & 60.00 & 6.00 & 100.00 & 45.00 & 55.00 & 0.81 \\
\hline KDM-AhJ-28 & 6.00 & 68.00 & 7.00 & 102.00 & 50.00 & 52.00 & 0.96 \\
\hline KDM-AhJ-29 & 7.00 & 75.00 & 5.00 & 67.00 & 37.00 & 30.00 & 1.23 \\
\hline KDM-AhJ-30 & 6.00 & 60.00 & 4.00 & 66.00 & 40.00 & 26.00 & 1.53 \\
\hline KDM-AhJ-31 & 3.20 & 72.00 & 4.80 & 67.00 & 40.00 & 27.00 & 1.48 \\
\hline KDM-AhJ-32 & 6.00 & 40.00 & 5.00 & 125.00 & 60.00 & 65.00 & 0.9 \\
\hline KDM-AhJ-33 & 4.00 & 40.00 & 4.00 & 100.00 & 60.00 & 40.00 & 1.50 \\
\hline KDM-AhJ-34 & 5.00 & 100.00 & 4.00 & 40.00 & 25.00 & 15.00 & 1.60 \\
\hline KDM-AhJ-35 & 6.00 & 150.00 & 6.00 & 40.00 & 30.00 & 10.00 & 3.00 \\
\hline KDM-AhJ-36 & 6.00 & 100.00 & 4.00 & 40.00 & 30.00 & 10.00 & 3.00 \\
\hline KDM-AhJ-37 & 5.00 & 150.00 & 3.20 & 20.00 & 12.00 & 8.00 & 1.50 \\
\hline KDM-AhJ-38 & 5.00 & 75.00 & 5.00 & 67.00 & 50.00 & 17.00 & 2.90 \\
\hline KDM-AhJ-39 & 4.50 & 150.00 & 8.00 & 55.00 & 30.00 & 25.00 & 1.20 \\
\hline KDM-AhJ-40 & 3.50 & 50.00 & 3.50 & 75.00 & 60.00 & 15.00 & 4.00 \\
\hline
\end{tabular}




\begin{tabular}{|c|c|c|c|c|c|c|c|}
\hline KDM-AhJ-41 & 4.70 & 78.00 & 4.30 & 55.00 & 30.00 & 25.00 & 1.20 \\
\hline KDM-AhJ-42 & 4.00 & 100.00 & 6.00 & 60.00 & 40.00 & 20.00 & 2.00 \\
\hline KDM-AhJ-43 & 3.00 & 75.00 & 6.00 & 80.00 & 70.00 & 10.00 & 7.00 \\
\hline KDM-AhJ-44 & 5.40 & 100.00 & 7.60 & 75.00 & 60.00 & 15.00 & 4.00 \\
\hline KDM-AhJ-45 & 6.60 & 52.00 & 4.40 & 85.00 & 60.00 & 25.00 & 2.40 \\
\hline KDM-AhJ-46 & 4.40 & 210.00 & 13.60 & 80.00 & 67.00 & 13.00 & 5.20 \\
\hline KDM-AhJ-47 & 6.50 & 75.00 & 5.50 & 80.00 & 55.00 & 25.00 & 2.20 \\
\hline KDM-AhJ-48 & 5.20 & 50.00 & 6.80 & 110.00 & 60.00 & 50.00 & 1.20 \\
\hline KDM-AhJ-49 & 6.90 & 42.00 & 7.10 & 170.00 & 110.00 & 60.00 & 1.80 \\
\hline KDM-AhJ-50 & 7.00 & 50.00 & 5.00 & 100.00 & 60.00 & 40.00 & 1.50 \\
\hline KDM-AhJ-51 & 5.00 & 60.00 & 4.00 & 67.00 & 45.00 & 17.00 & 2.60 \\
\hline KDM-AhJ-52 & 6.50 & 50.00 & 5.50 & 110.00 & 65.00 & 45.00 & 1.40 \\
\hline KDM-AhJ-53 & 4.70 & 62.00 & 4.30 & 70.00 & 55.00 & 15.00 & 3.60 \\
\hline KDM-AhJ-54 & 6.00 & 75.00 & 6.00 & 80.00 & 65.00 & 15.00 & 4.30 \\
\hline KDM-AhJ- 55 & 5.50 & 50.00 & 4.50 & 90.00 & 75.00 & 25.00 & 3.00 \\
\hline Max. & 8.00 & 210.00 & 13.60 & 170.00 & 110.00 & 65.00 & 7.00 \\
\hline Min. & 3.00 & 30.00 & 3.00 & 20.00 & 12.00 & 8.00 & 0.42 \\
\hline Mean & 5.38 & 81.00 & 5.20 & 71.29 & 46.16 & 25.24 & 2.28 \\
\hline SD & 1.18 & 35.77 & 1.89 & 27.34 & 17.51 & 15.10 & 1.30 \\
\hline SE(+) & 0.43 & 2.30 & 0.30 & 2.70 & 2.56 & 1.20 & 0.11 \\
\hline CV(\%) & 1.37 & 1256.36 & 3.52 & 734.09 & 301.08 & 223.96 & 1.66 \\
\hline
\end{tabular}

Table.4 Score card for evaluation of Qualitative Characters of Jackfruit Germplasm

\begin{tabular}{|c|c|c|c|c|c|}
\hline $\begin{array}{c}\text { Acc. } \\
\text { No. }\end{array}$ & $\begin{array}{c}\text { Colour and } \\
\text { appearance }\end{array}$ & Flavor & $\begin{array}{c}\text { Texture/Fir } \\
\text { mness }\end{array}$ & Taste & $\begin{array}{c}\text { Overall } \\
\text { acceptability }\end{array}$ \\
\hline KDM-AhJ-1 & 7.60 & 6.80 & 7.20 & 7.10 & 7.80 \\
\hline KDM-AhJ-2 & 7.20 & 6.20 & 6.20 & 5.00 & 5.20 \\
\hline KDM-AhJ-3 & 6.40 & 5.90 & 6.60 & 6.50 & 5.70 \\
\hline KDM-AhJ-4 & 6.70 & 5.70 & 5.90 & 5.80 & 7.80 \\
\hline KDM-AhJ-5 & 6.70 & 7.00 & 6.10 & 6.40 & 6.30 \\
\hline KDM-AhJ-6 & 6.30 & 6.00 & 6.00 & 4.90 & 5.30 \\
\hline KDM-AhJ-7 & 7.90 & 7.50 & 7.70 & 8.10 & 7.50 \\
\hline KDM-AhJ-8 & 6.90 & 7.00 & 8.60 & 7.60 & 9.10 \\
\hline KDM-Ah-9 & 8.00 & 8.00 & 8.00 & 8.00 & 7.50 \\
\hline KDM-AhJ-10 & 9.00 & 9.00 & 9.00 & 9.00 & 8.30 \\
\hline KDM-AhJ-11 & 7.30 & 6.60 & 6.80 & 8.30 & 6.90 \\
\hline KDM-AhJ-12 & 6.60 & 7.10 & 7.70 & 7.10 & 5.10 \\
\hline KDM-AhJ-13 & 7.60 & 7.40 & 7.10 & 8.00 & 7.40 \\
\hline KDM-AhJ-14 & 6.80 & 6.70 & 7.00 & 6.30 & 5.70 \\
\hline KDM-AhJ-15 & 7.00 & 7.30 & 6.90 & 7.20 & 5.90 \\
\hline KDM-AhJ-16 & 5.30 & 6.40 & 6.10 & 5.90 & 5.80 \\
\hline KDM-AhJ-17 & 7.10 & 6.80 & 6.60 & 6.40 & 5.60 \\
\hline KDM-AhJ-18 & 5.20 & 5.70 & 6.10 & 6.50 & 6.20 \\
\hline KDM-AhJ-19 & 6.00 & 5.40 & 5.70 & & \\
\hline
\end{tabular}




\begin{tabular}{|c|c|c|c|c|c|}
\hline KDM-AhJ-20 & 7.00 & 7.20 & 7.50 & 7.10 & 6.90 \\
\hline KDM-AhJ-21 & 7.10 & 7.30 & 7.00 & 6.90 & 6.70 \\
\hline KDM-AhJ-22 & 5.90 & 5.70 & 6.10 & 6.10 & 5.60 \\
\hline KDM-AhJ-23 & 6.10 & 6.50 & 6.20 & 5.70 & 6.80 \\
\hline KDM-AhJ-24 & 6.20 & 5.40 & 6.10 & 5.30 & 5.80 \\
\hline KDM-AhJ-25 & 5.80 & 6.10 & 6.20 & 5.70 & 5.80 \\
\hline KDM-AhJ-26 & 6.10 & 5.70 & 5.50 & 5.80 & 6.20 \\
\hline KDM-AhJ-27 & 7.20 & 6.90 & 5.90 & 6.30 & 6.40 \\
\hline KDM-AhJ-28 & 6.40 & 6.80 & 7.10 & 7.30 & 6.70 \\
\hline KDM-AhJ-29 & 7.30 & 7.80 & 7.10 & 6.90 & 6.00 \\
\hline KDM-AhJ-30 & 5.30 & 5.70 & 6.20 & 5.40 & 5.50 \\
\hline KDM-AhJ-31 & 6.30 & 6.50 & 6.10 & 6.80 & 8.50 \\
\hline KDM-AhJ-32 & 6.20 & 6.40 & 5.30 & 6.10 & 9.00 \\
\hline KDM-AhJ-33 & 7.40 & 7.60 & 7.10 & 7.50 & 6.20 \\
\hline KDM-AhJ-34 & 6.90 & 5.90 & 6.10 & 7.10 & 8.10 \\
\hline KDM-AhJ-35 & 7.80 & 7.10 & 7.90 & 7.70 & 8.50 \\
\hline KDM-AhJ-36 & 6.00 & 4.00 & 6.00 & 4.00 & 5.00 \\
\hline KDM-AhJ-37 & 5.00 & 5.00 & 4.00 & 3.00 & 6.00 \\
\hline KDM-AhJ-38 & 6.00 & 5.00 & 4.00 & 4.00 & 7.00 \\
\hline KDM-AhJ-39 & 5.00 & 4.00 & 5.00 & 6.00 & 5.20 \\
\hline KDM-AhJ-40 & 6.00 & 5.00 & 4.00 & 6.00 & 7.00 \\
\hline KDM-AhJ-41 & 6.00 & 6.00 & 7.00 & 6.00 & 8.00 \\
\hline KDM-AhJ-42 & 5.00 & 5.00 & 6.00 & 5.00 & 8.00 \\
\hline KDM-AhJ-43 & 4.00 & 5.00 & 5.00 & 5.00 & 6.00 \\
\hline KDM-AhJ-44 & 5.00 & 5.00 & 6.00 & 6.00 & 6.00 \\
\hline KDM-AhJ-45 & 6.00 & 5.00 & 7.00 & 6.00 & 7.00 \\
\hline KDM-AhJ-46 & 9.00 & 8.50 & 8.50 & 9.00 & 9.20 \\
\hline KDM-AhJ-47 & 5.00 & 6.00 & 5.00 & 5.00 & 6.00 \\
\hline KDM-AhJ-48 & 4.00 & 5.00 & 4.00 & 5.00 & 5.30 \\
\hline KDM-AhJ-49 & 5.00 & 6.00 & 5.00 & 4.00 & 5.70 \\
\hline KDM-AhJ-50 & 5.00 & 6.00 & 7.00 & 5.00 & 5.30 \\
\hline KDM-AhJ-51 & 5.00 & 7.00 & 5.00 & 6.00 & 5.00 \\
\hline KDM-AhJ-52 & 6.00 & 5.00 & 4.00 & 5.00 & 6.00 \\
\hline KDM-AhJ-53 & 6.00 & 5.00 & 6.00 & 5.00 & 6.00 \\
\hline KDM-AhJ-54 & 5.00 & 5.00 & 6.00 & 7.00 & 6.00 \\
\hline KDM-AhJ- 55 & 6.00 & 6.00 & 7.00 & 5.00 & 7.00 \\
\hline Max. & 9.00 & 9.00 & 9.00 & 9.00 & 9.30 \\
\hline Min. & 4.00 & 4.00 & 4.00 & 3.00 & 5.00 \\
\hline Mean & 6.30 & 6.21 & 6.31 & 6.21 & 6.66 \\
\hline SD & 1.09 & 1.06 & 1.2 & 1.29 & 1.18 \\
\hline $\operatorname{SE}( \pm)$ & 0.31 & 0.24 & 0.25 & 0.23 & 0.27 \\
\hline CV (\%) & 1.08 & 1.05 & 1.34 & 1.57 & 1.33 \\
\hline
\end{tabular}

The above classifications were based on the descriptor developed for Artocarpus heterophyllus Lam. (Jackfruit) compiled by International Plant Genetic Resources Institute (IPGRI, 2000) and the scores were derived from the average of twenty participants 
Table.5 Quality characters of jackfruit genotypes KDM Ah1 - KDM Ah55 (2018-2019)

\begin{tabular}{|c|c|c|c|}
\hline $\begin{array}{l}\text { Acc. } \\
\text { No. }\end{array}$ & $\begin{array}{l}\text { Fruit } \\
\text { shape }\end{array}$ & Flakes colour & Shelf life of flakes \\
\hline KDM-AhJ-1 & slender & Yellow & One day \\
\hline KDM-AhJ-2 & slender & Light yellow & One day \\
\hline KDM-AhJ-3 & Round/oval & Light yellow & One day \\
\hline KDM-AhJ-4 & slender & yellow & One day \\
\hline KDM-AhJ-5 & oval & Light yellow & One day \\
\hline KDM-AhJ-6 & round & white & One day \\
\hline KDM-AhJ-7 & Oval & Bright yellow & One day \\
\hline KDM-AhJ-8 & Slender & Light yellow & Three days \\
\hline KDM-Ah-9 & Slender & Yellow & One day \\
\hline KDM-AhJ-10 & Slender & Bright Yellow & Five days \\
\hline KDM-AhJ-11 & Round & Yellow & One day \\
\hline KDM-AhJ-12 & Round & yellow & One day \\
\hline KDM-AhJ-13 & Round & yellow & one day \\
\hline KDM-AhJ-14 & Round & Light yellow & One day \\
\hline KDM-AhJ-15 & Slender & Yellow & One day \\
\hline KDM-AhJ-16 & Slender & Pure white & One day \\
\hline KDM-AhJ-17 & Slender & Yellow & One day \\
\hline KDM-AhJ-18 & Slender & Pure White & One day \\
\hline KDM-AhJ-19 & Slender & Pure white & One day \\
\hline KDM-AhJ-20 & Round & White & One day \\
\hline KDM-AhJ-21 & Round & Light yellow & Half day \\
\hline KDM-AhJ-22 & Round & Light yellow & One day \\
\hline KDM-AhJ-23 & Slender & White & Half day \\
\hline KDM-AhJ-24 & Slender & White & One day \\
\hline KDM-AhJ-25 & Round & Light Yellow & One day \\
\hline KDM-AhJ-26 & Round & Light yellow & One day \\
\hline KDM-AhJ-27 & Round & Yellow & Two days \\
\hline KDM-AhJ-28 & Round & Yellow & Two days \\
\hline KDM-AhJ-29 & Slender & yellow & Two days \\
\hline KDM-AhJ-30 & Round & Light yellow & two days \\
\hline KDM-AhJ-31 & Slender & Light Yellow & One day \\
\hline KDM-AhJ-32 & Slender & Bright yellow & Two days \\
\hline KDM-AhJ-33 & Round & Yellow & One day \\
\hline KDM-AhJ-34 & Slender & Yellow & One day \\
\hline KDM-AhJ-35 & Slender & Bright yellow & Three days \\
\hline KDM-AhJ-36 & Oval & Light yellow & One day \\
\hline KDM-AhJ-37 & Slender & Light yellow & One day \\
\hline KDM-AhJ-38 & Slender & Yellow & One day \\
\hline KDM-AhJ-39 & Round & yellow & one day \\
\hline
\end{tabular}




\begin{tabular}{|l|l|l|l|}
\hline KDM-AhJ-40 & Round & Yellow & One day \\
\hline KDM-AhJ-41 & Slender & yellow & One day \\
\hline KDM-AhJ-42 & Slender & yellow & One day \\
\hline KDM-AhJ-43 & Slender & Light yellow & One day \\
\hline KDM-AhJ-44 & Round & Light yellow & One day \\
\hline KDM-AhJ-45 & Round & Light yellow & One day \\
\hline KDM-AhJ-46 & Slender & Bright Yellow & Five days \\
\hline KDM-AhJ-47 & Round & Light yellow & One day \\
\hline KDM-AhJ-48 & Oval & Light yellow & One day \\
\hline KDM-AhJ-49 & Oval & Light yellow & One day \\
\hline KDM-AhJ-50 & Round & yellow & One day \\
\hline KDM-AhJ-51 & Round & yellow & One day \\
\hline KDM-AhJ-52 & Round & Bright yellow & One day \\
\hline KDM-AhJ-53 & Round & yellow & One day \\
\hline KDM-AhJ-54 & Round & yellow & Two days \\
\hline KDM-AhJ-55 & Slender & Light yellow & Two days \\
\hline
\end{tabular}

Table.6 Rainfall (mm) of Pudukkottai District (Alangudi and Thiruvarankulam Taluk) of Tamil Nadu (2016-2019)

\begin{tabular}{|l|l|l|l|l|}
\hline Months & $\mathbf{2 0 1 6}$ & $\mathbf{2 0 1 7}$ & $\mathbf{2 0 1 8}$ & $\mathbf{2 0 1 9}$ \\
\hline January & 0.00 & 36.71 & 0.00 & - \\
\hline February & 0.00 & 1.45 & 0.00 & - \\
\hline March & 0.00 & 37.04 & 0.00 & - \\
\hline April & 2.96 & 0.00 & 0.00 & 31.20 \\
\hline May & 78.27 & 0.00 & 10.50 & 5.80 \\
\hline June & 30.48 & 15.12 & 10.60 & 41.60 \\
\hline July & 55.23 & 20.45 & 8.10 & 79.00 \\
\hline August & 75.78 & 15.23 & 10.50 & 13.40 \\
\hline September & 75.35 & 20.27 & 69.20 & 301.00 \\
\hline October & 79.27 & 17.25 & 115.80 & 205.80 \\
\hline November & 26.05 & 15.10 & 243.00 & - \\
\hline December & 73.23 & 10.25 & 29.40 & \\
\hline $\begin{array}{l}\text { Average } \\
\text { annual rainfall } \\
\text { (mm) }\end{array}$ & $\mathbf{4 9 6 . 6 2}$ & $\mathbf{1 8 8 . 8 7}$ & $\mathbf{4 9 7 . 1 0}$ & $\mathbf{6 7 7 . 8 0}$ \\
\hline
\end{tabular}


Table.7 Temperature of Pudukkottai District (Alangudi and Thiruvarankulam Taluk) of Tamil Nadu (2016-2019)

\begin{tabular}{|l|l|l|l|l|}
\hline Months & $\mathbf{2 0 1 6}$ & $\mathbf{2 0 1 7}$ & $\mathbf{2 0 1 8}$ & $\mathbf{2 0 1 9}$ \\
\hline January & 30 & 32 & 32 & 32 \\
\hline February & 29 & 33 & 32 & 34 \\
\hline March & 32 & 32 & 32 & 36 \\
\hline April & 33 & 34 & 34 & 38 \\
\hline May & 34 & 35 & 35 & 39 \\
\hline June & 33 & 34 & 30 & 32 \\
\hline July & 30 & 32 & 32 & 34 \\
\hline August & 28 & 30 & 28 & 32 \\
\hline September & 28 & 32 & 29 & 30 \\
\hline October & 26 & 32 & 32 & 32 \\
\hline November & 26 & 30 & 30 & 28 \\
\hline December & 26 & 28 & 25 & - \\
\hline
\end{tabular}
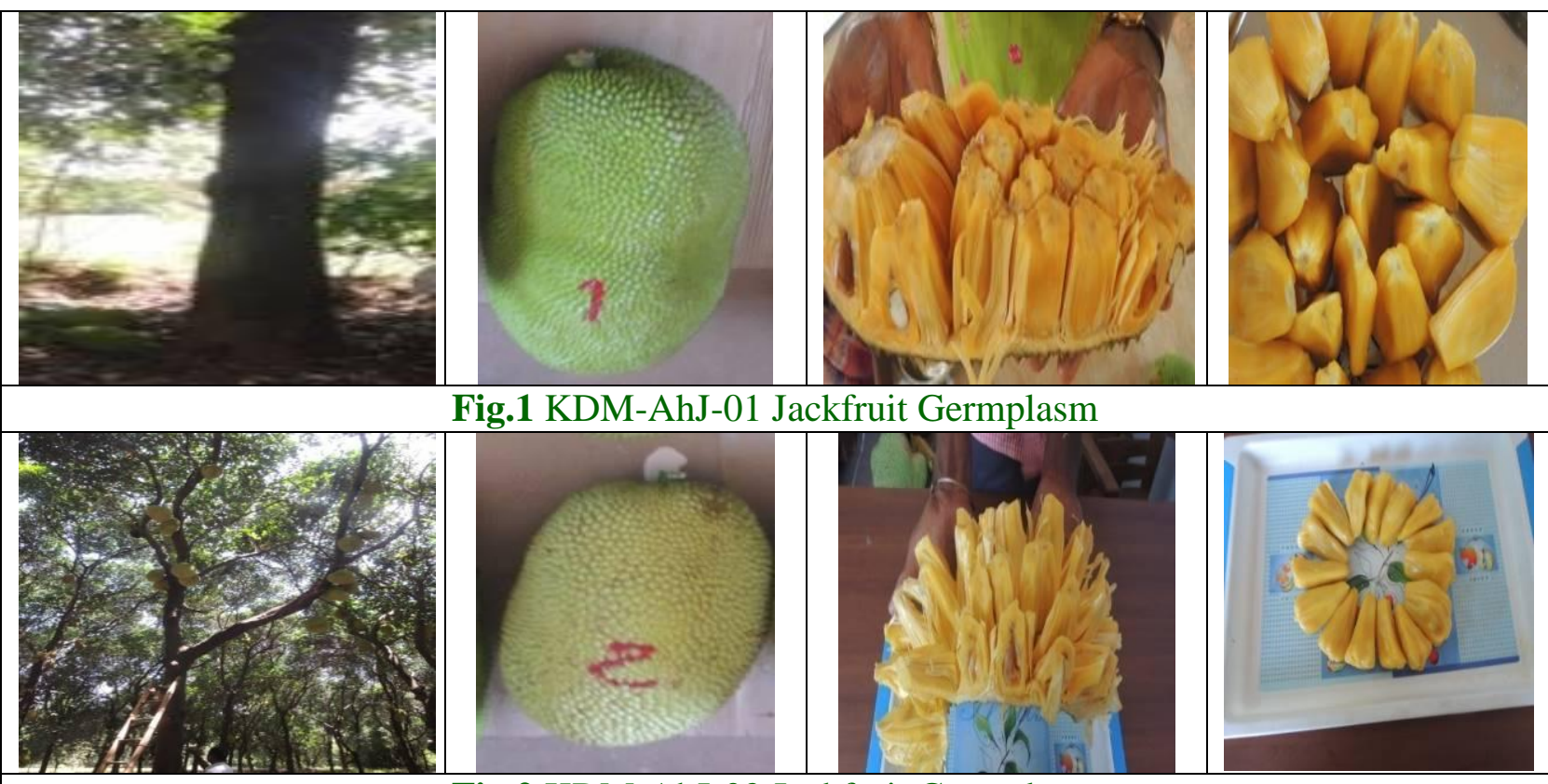

Fig.1 KDM-AhJ-01 Jackfruit Germplasm

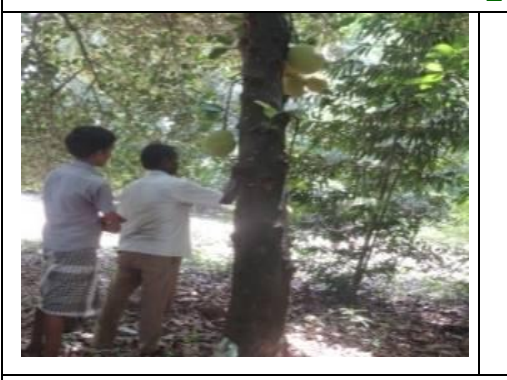

Fig.2 KDM-AhJ-02 Jackfruit Germplasm
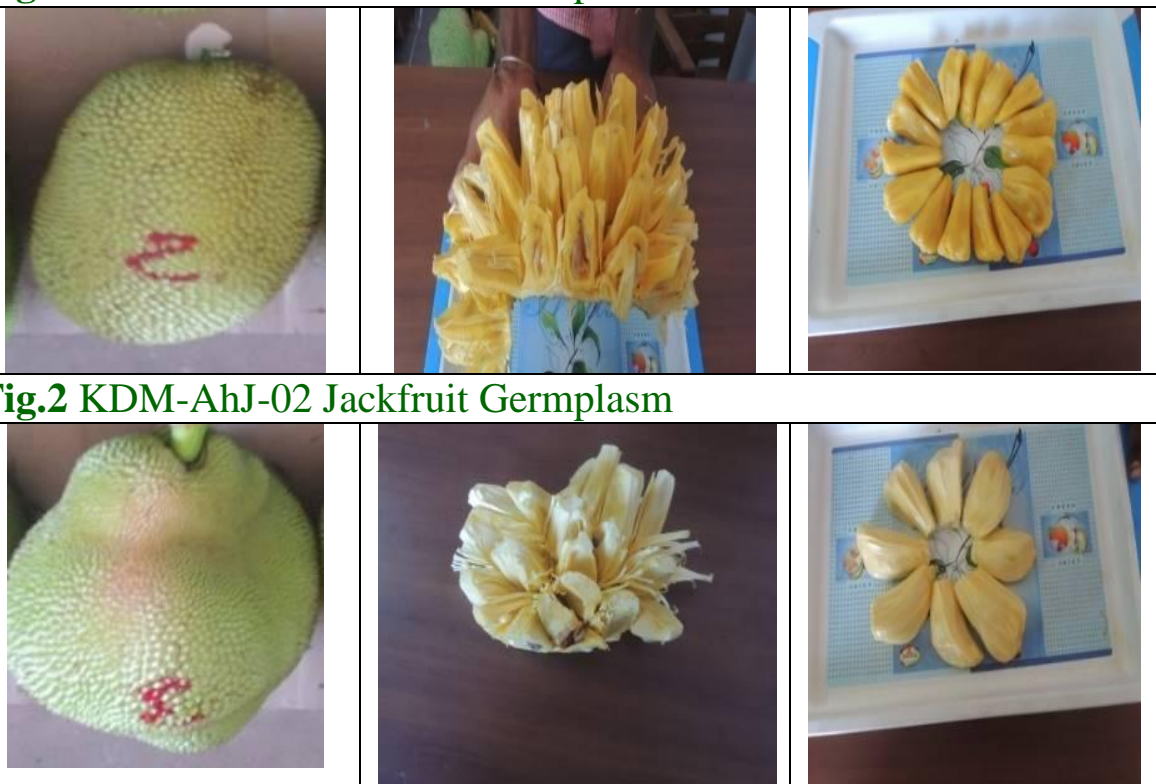

Fig.3 KDM-AhJ-03 Jackfruit Germplasm

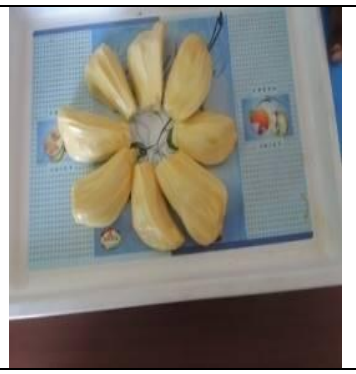



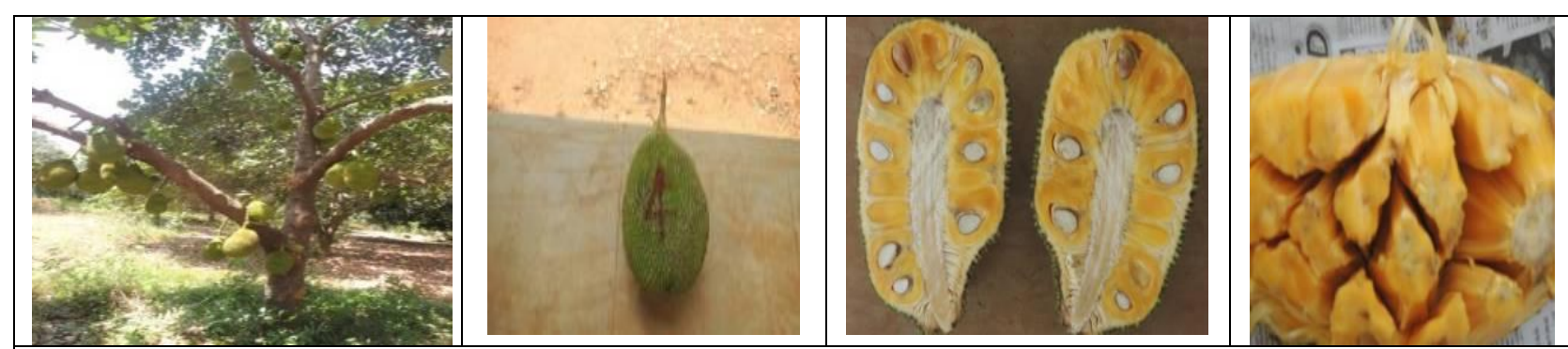

Fig.4 KDM-AhJ-04 Jackfruit Germplasm
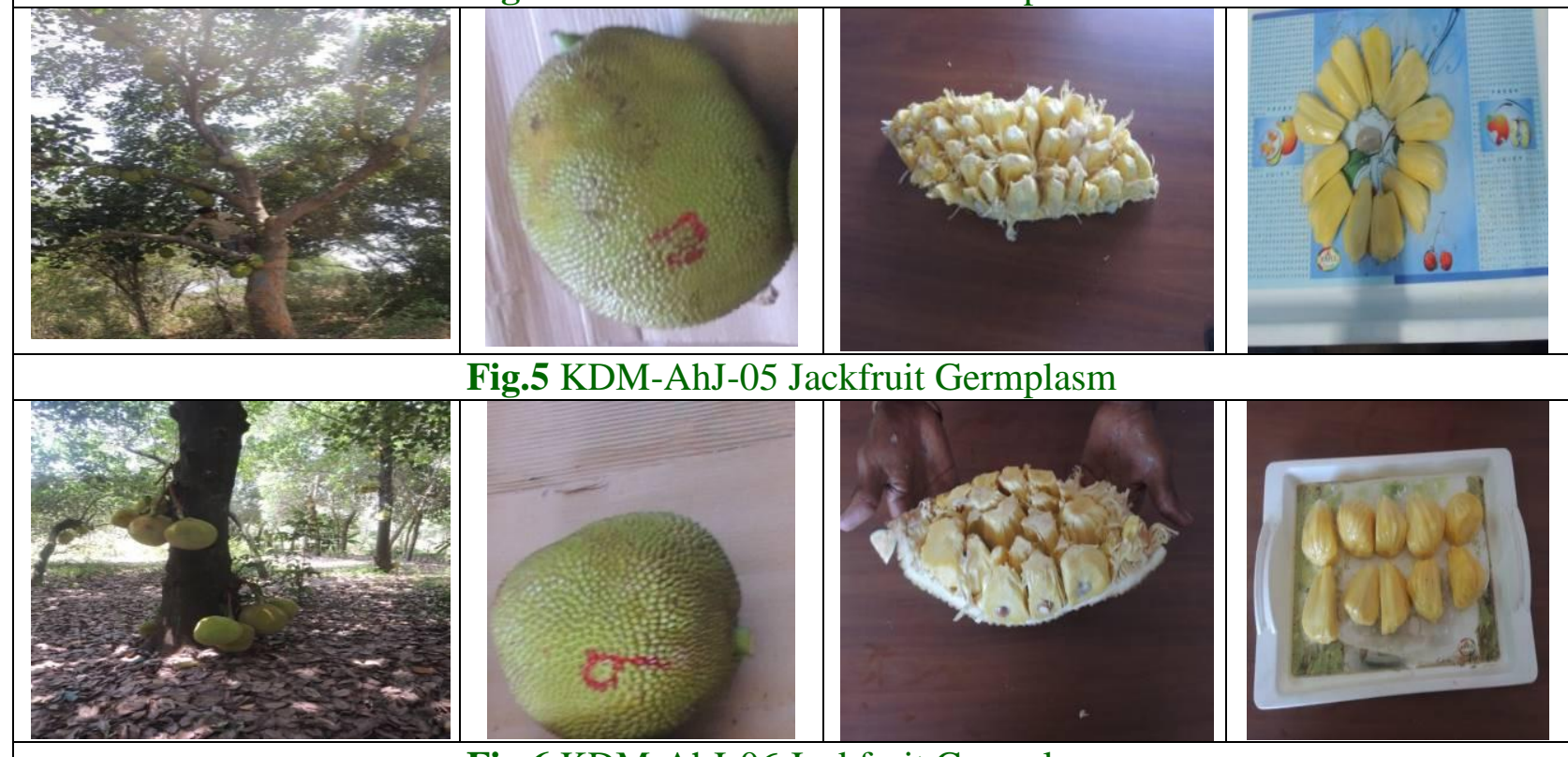

Fig.6 KDM-AhJ-06 Jackfruit Germplasm
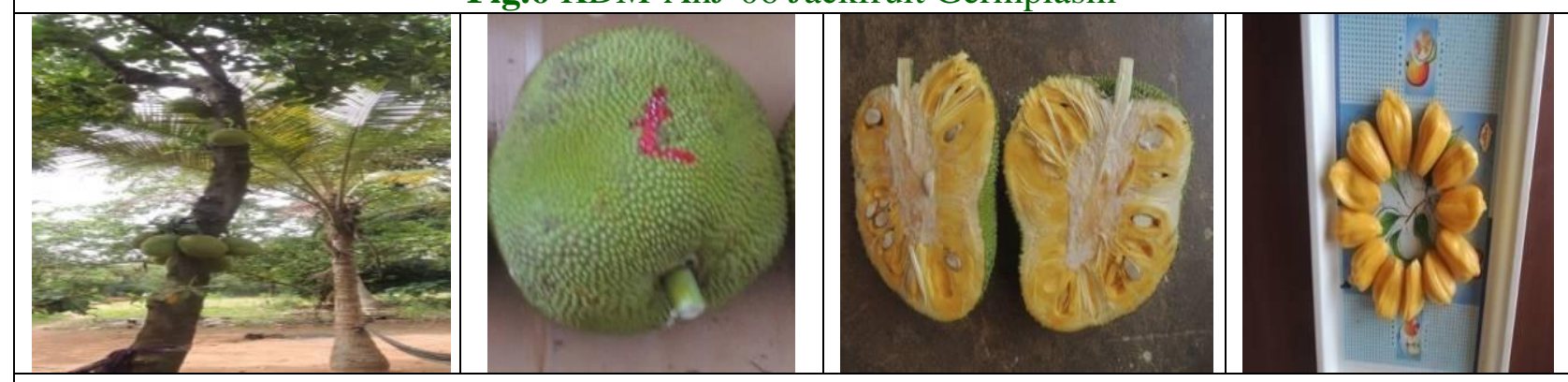

Fig.7 KDM-AhJ-07 Jackfruit Germplasm
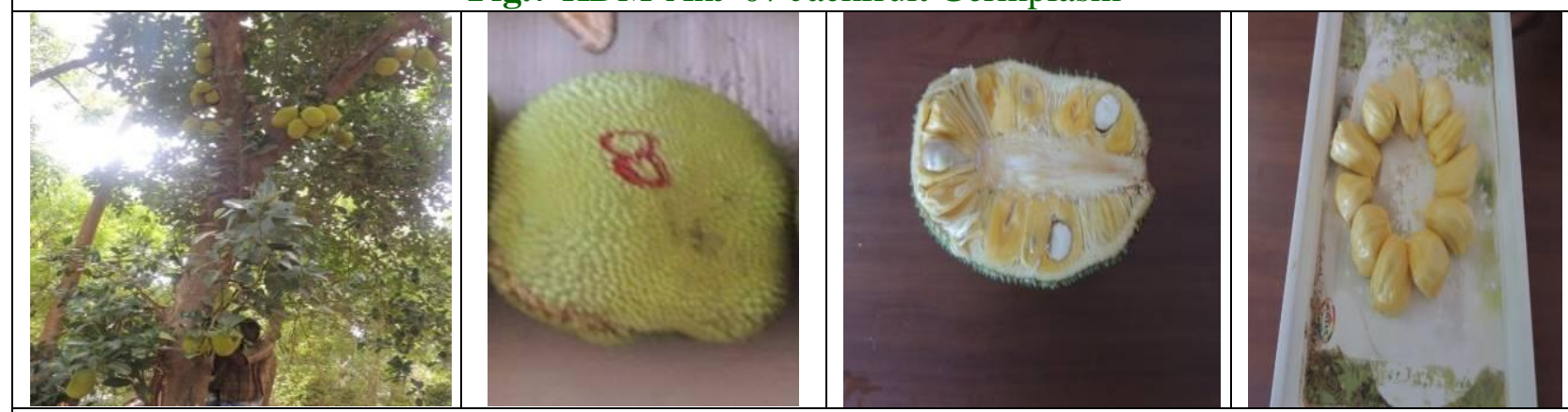

Fig.8 KDM-AhJ-08 Jackfruit Germplasm 


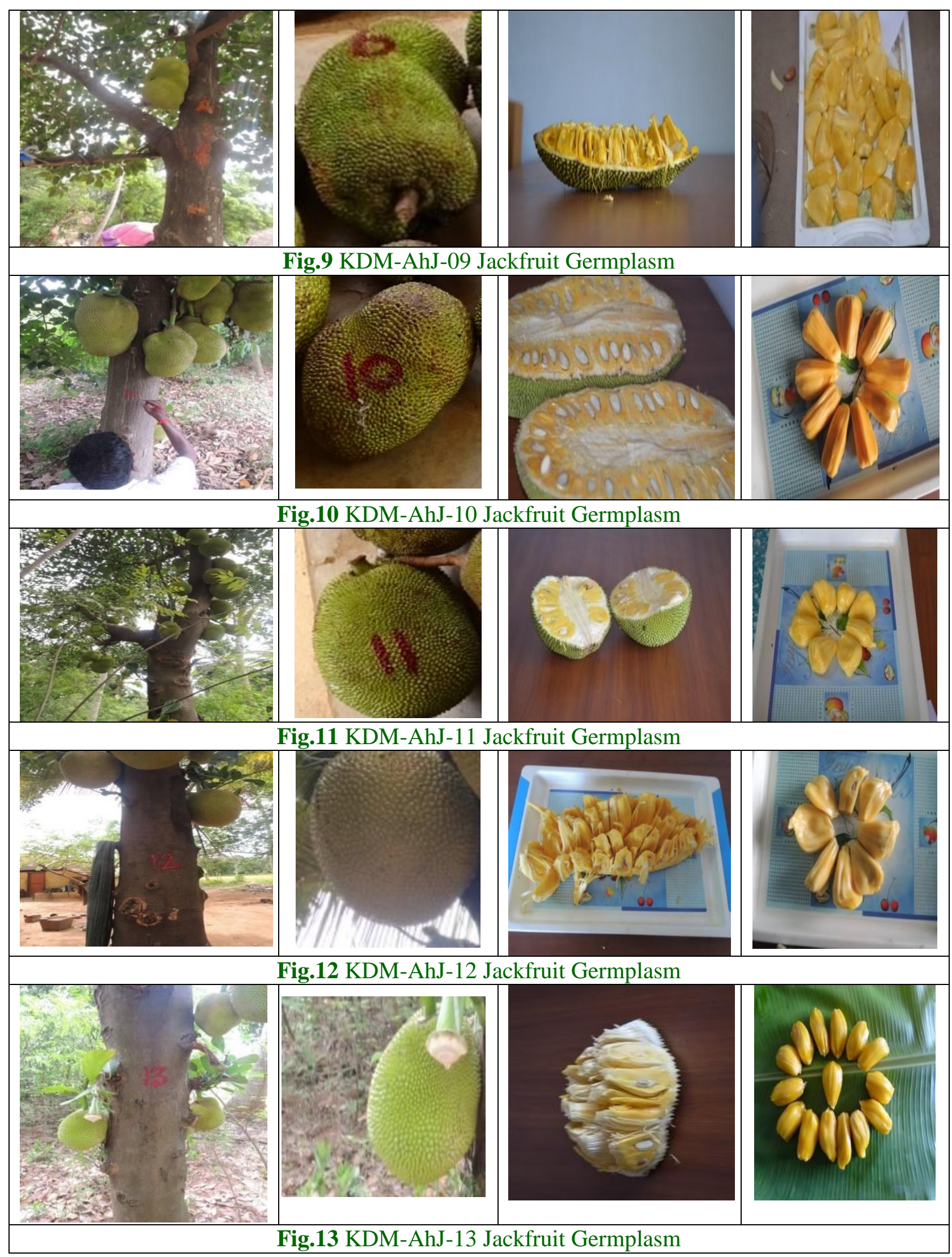



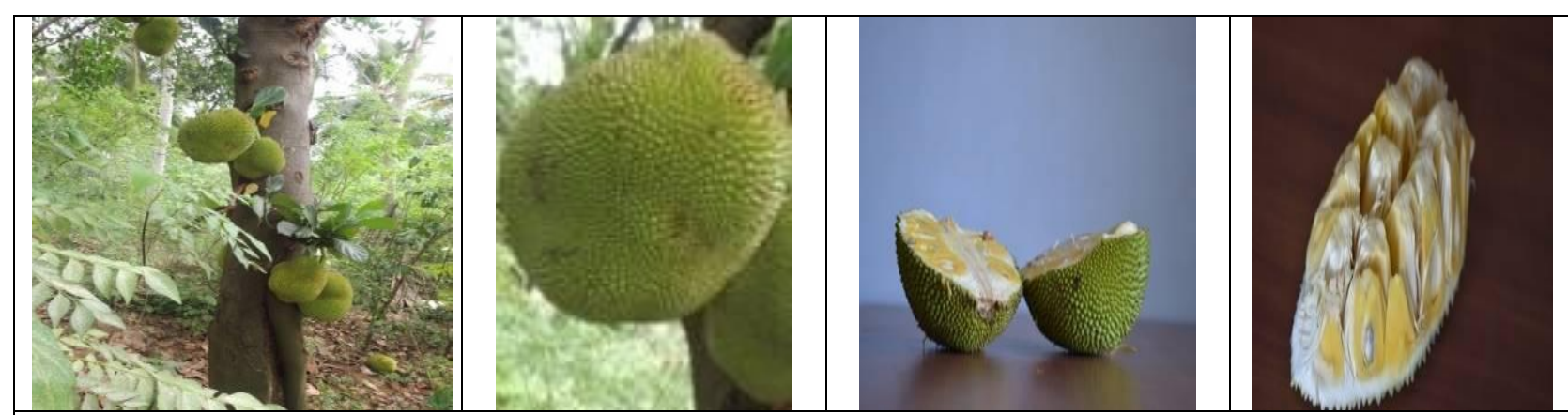

Fig.14 KDM-AhJ-14 Jackfruit Germplasm
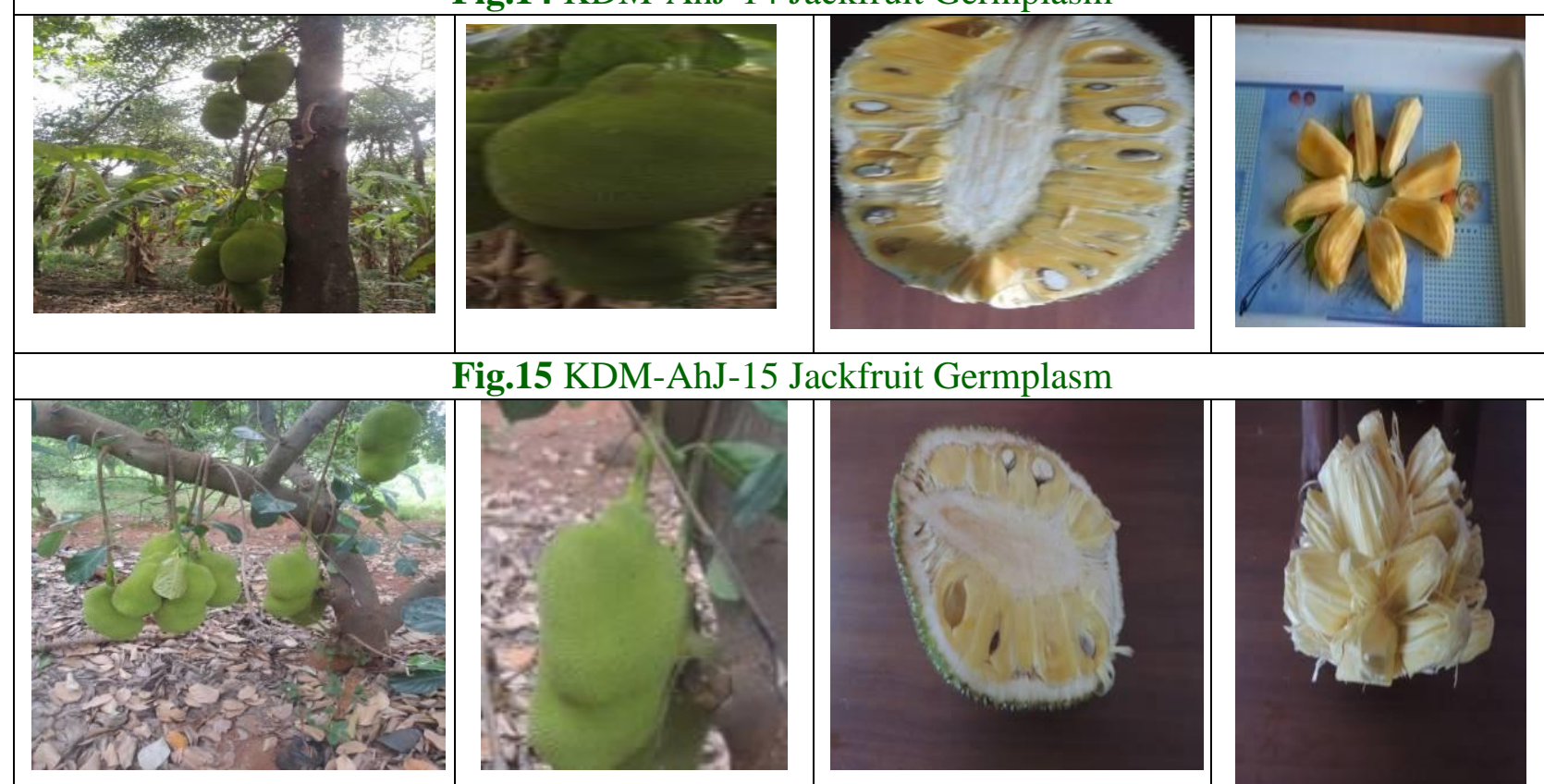

Fig.15 KDM-AhJ-15 Jackfruit Germplasm
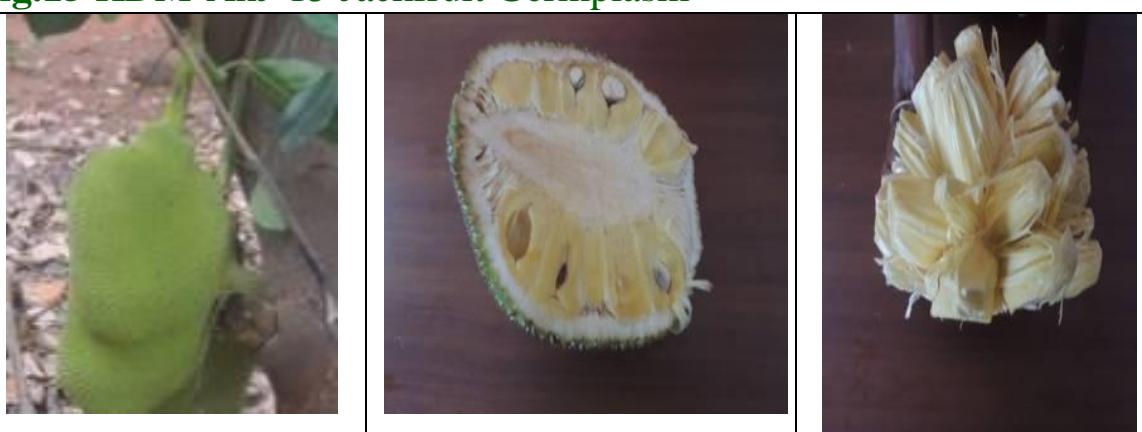

Fig.16 KDM-AhJ-16 Jackfruit Germplasm
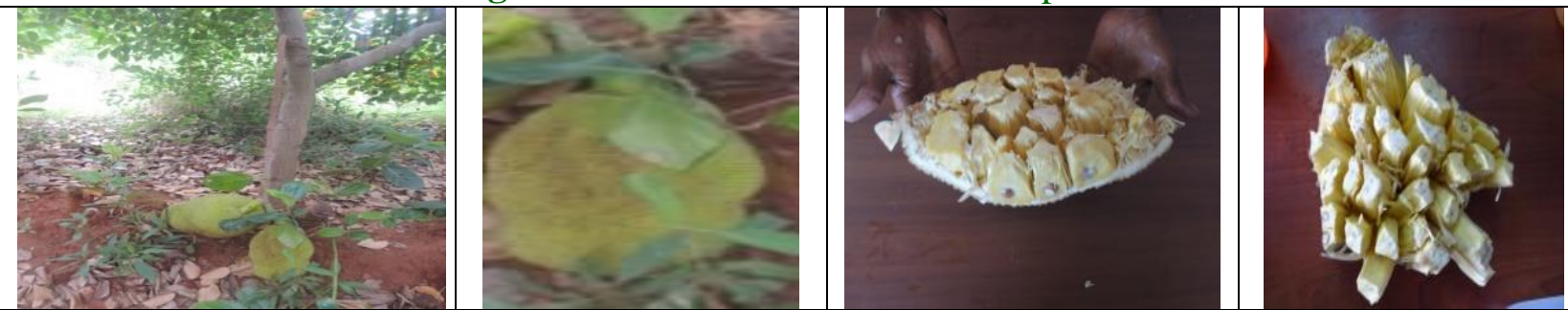

Fig.17 KDM-AhJ-17 Jackfruit Germplasm
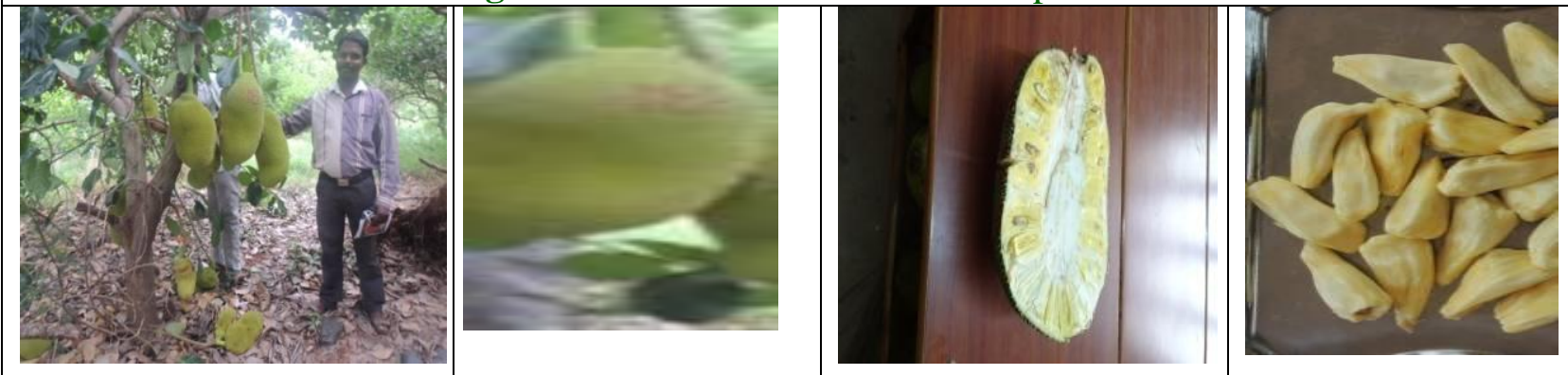

Fig.18 KDM-AhJ-18 Jackfruit Germplasm 


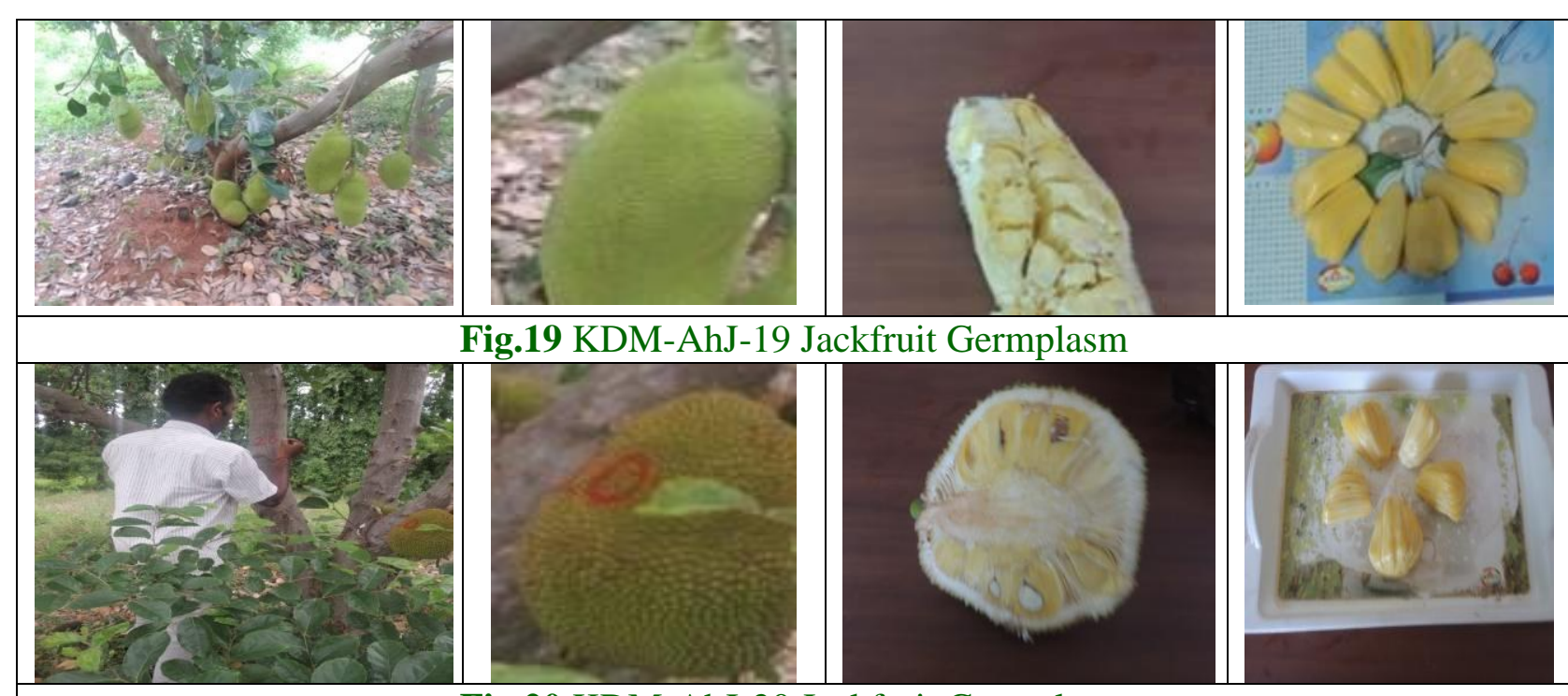

Fig.20 KDM-AhJ-20 Jackfruit Germplasm

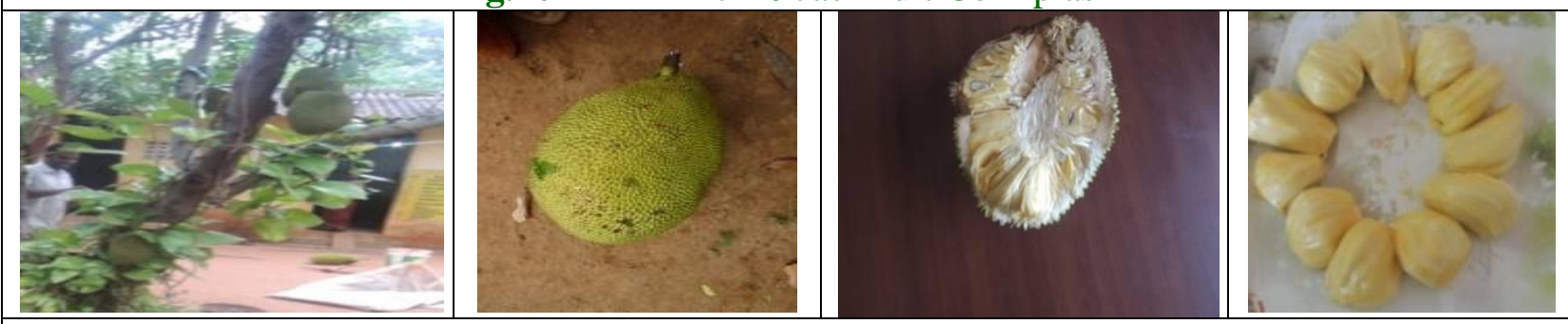

Fig.21 KDM-AhJ-21 Jackfruit Germplasm

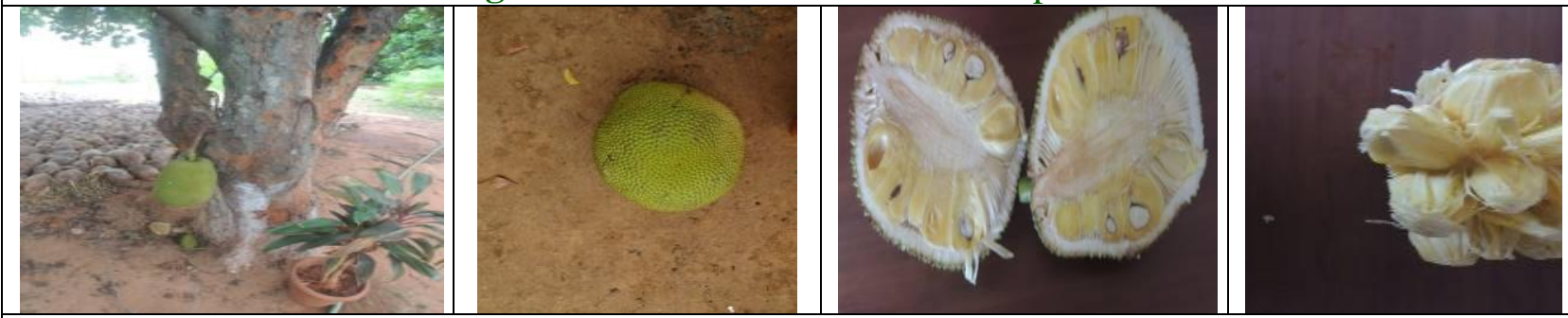

Fig.22 KDM-AhJ-22 Jackfruit Germplasm
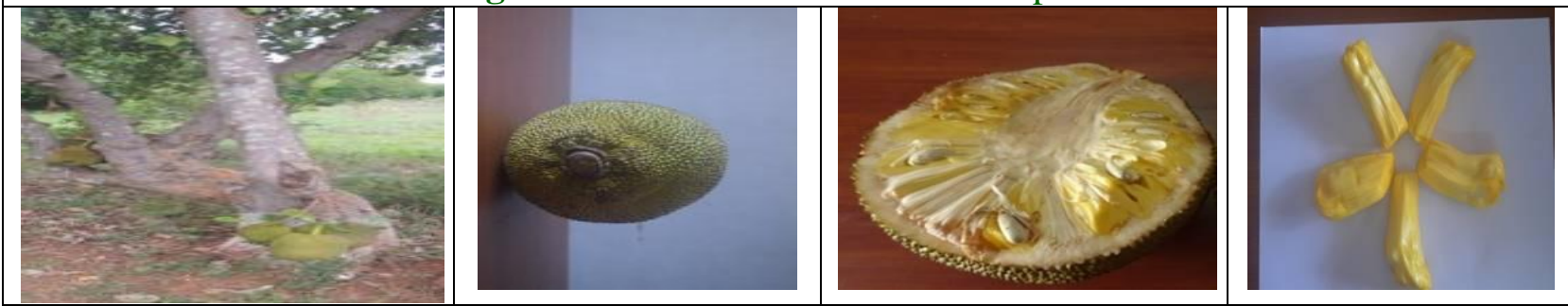

Fig.23 KDM-AhJ-23 Jackfruit Germplasm
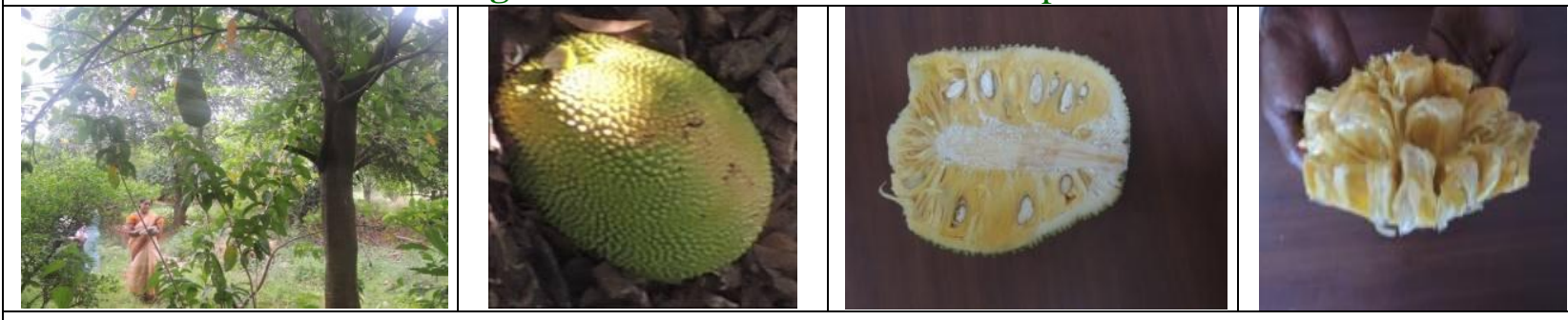

Fig.24 KDM-AhJ-24 Jackfruit Germplasm 

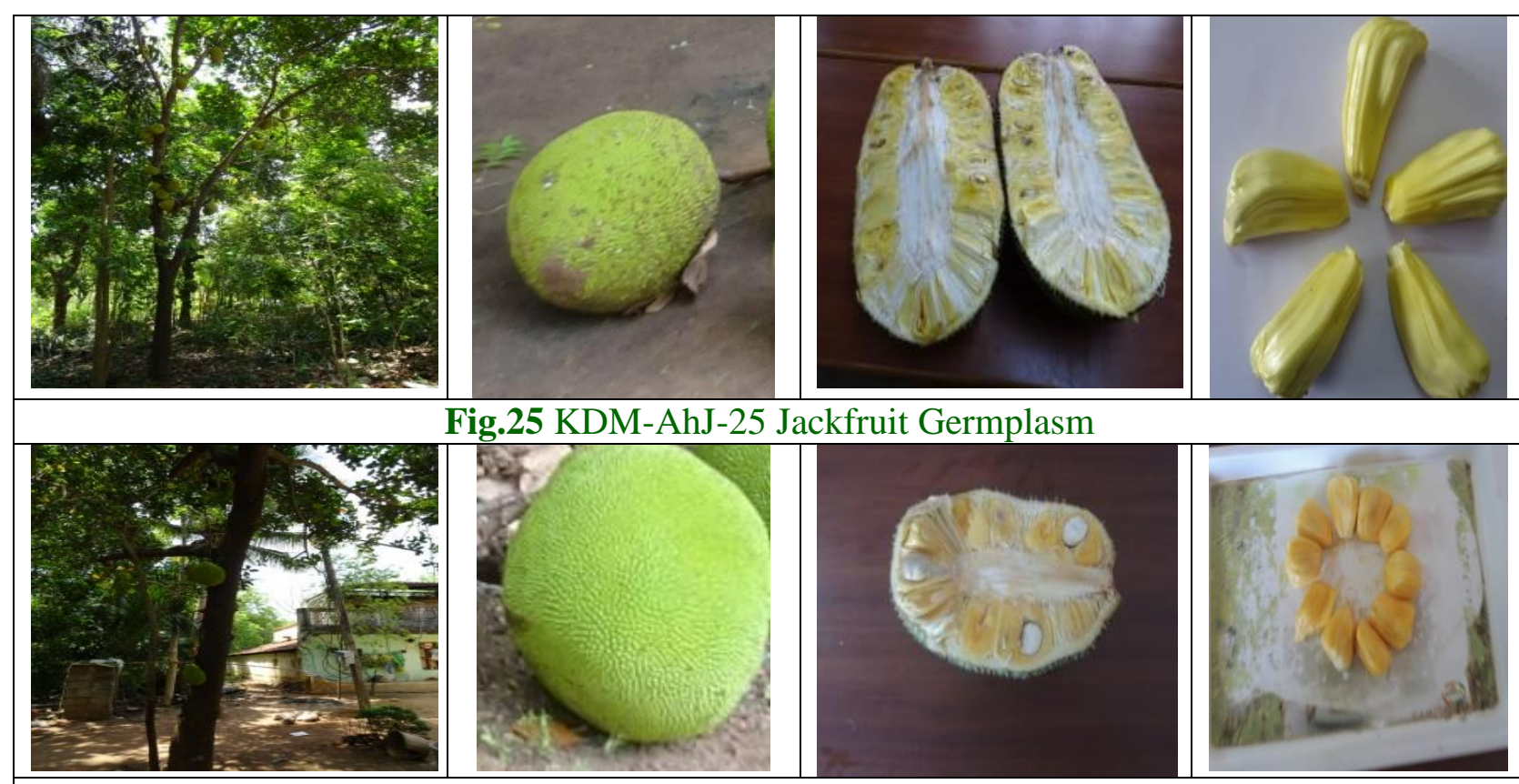

Fig.25 KDM-AhJ-25 Jackfruit Germplasm
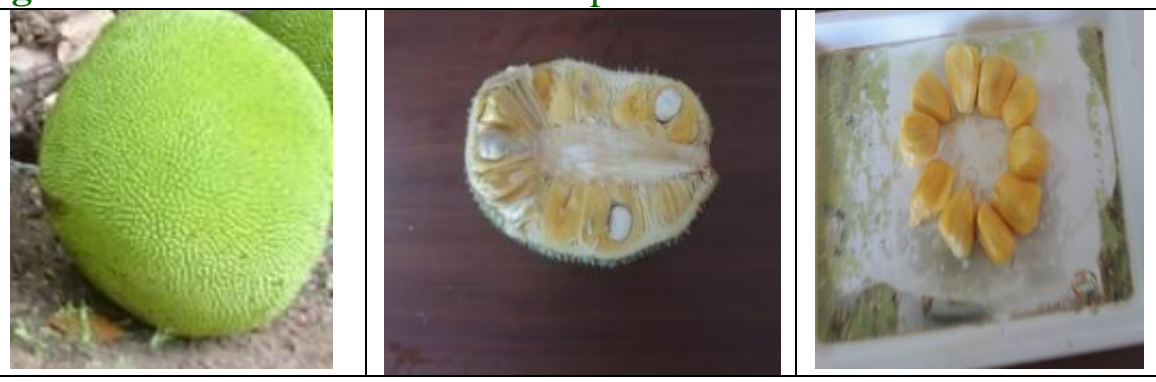

Fig.26 KDM-AhJ-26 Jackfruit Germplasm
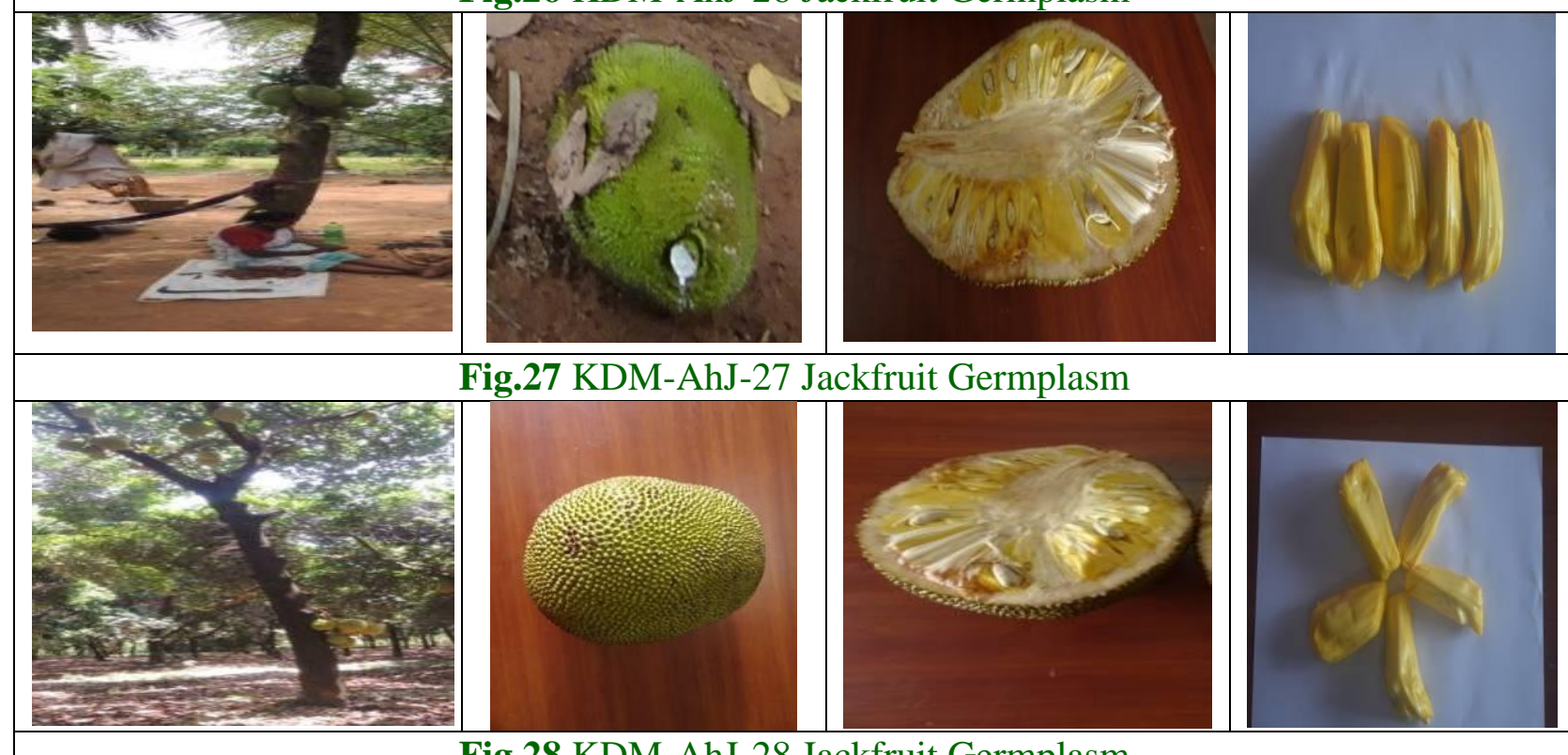

Fig.27 KDM-AhJ-27 Jackfruit Germplasm

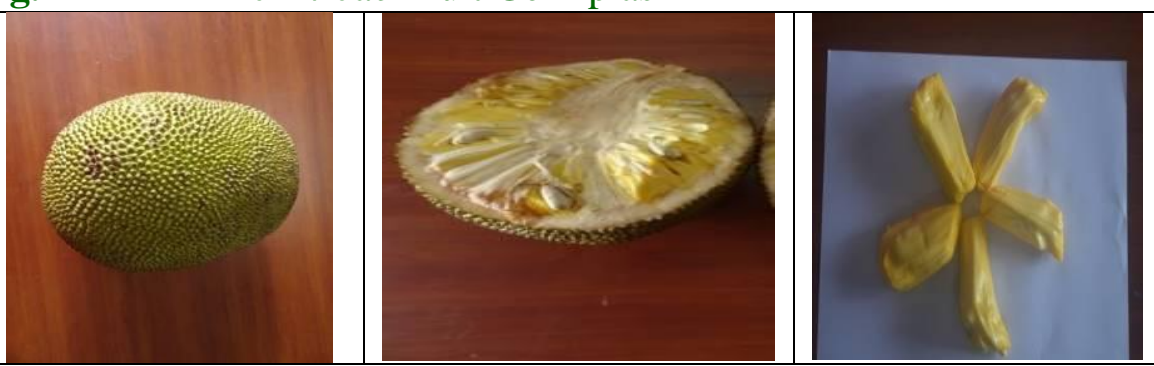

Fig.28 KDM-AhJ-28 Jackfruit Germplasm

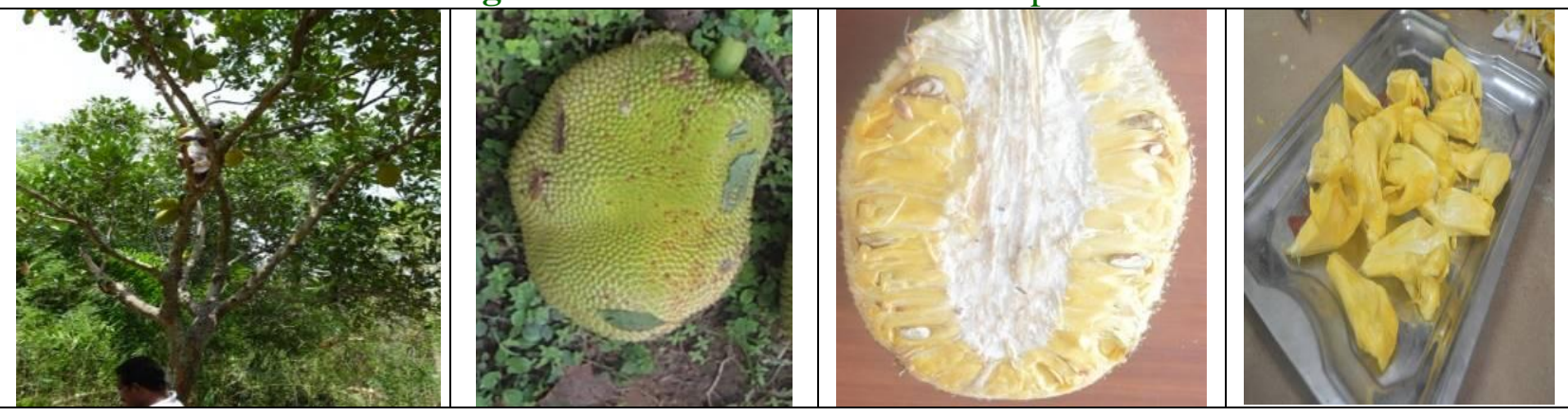

Fig.29 KDM-AhJ-29 Jackfruit Germplasm 

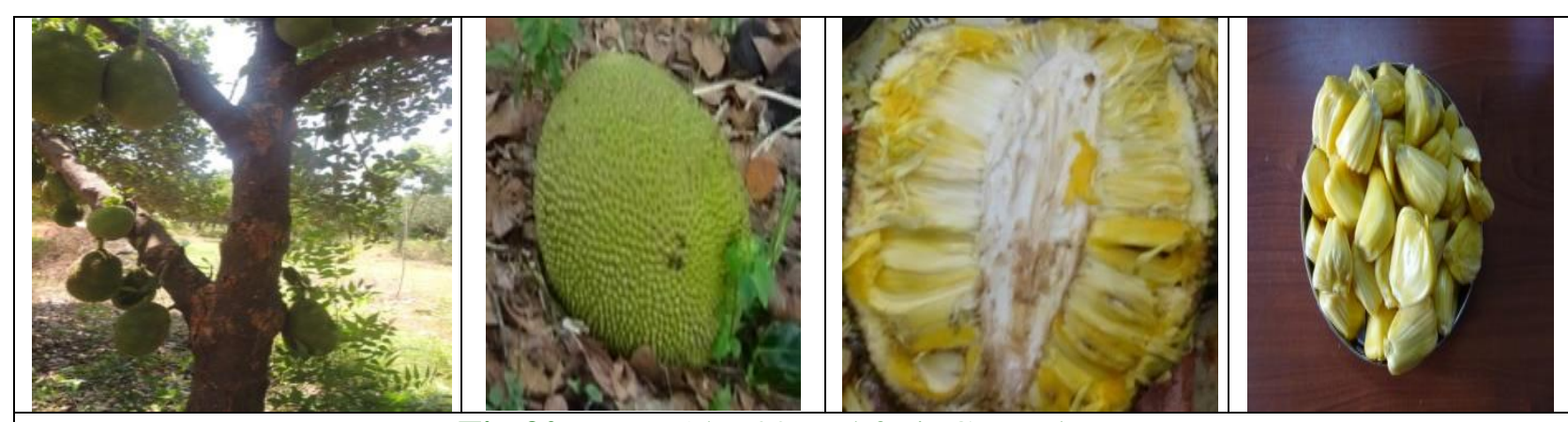

Fig.30 KDM-AhJ-30 Jackfruit Germplasm
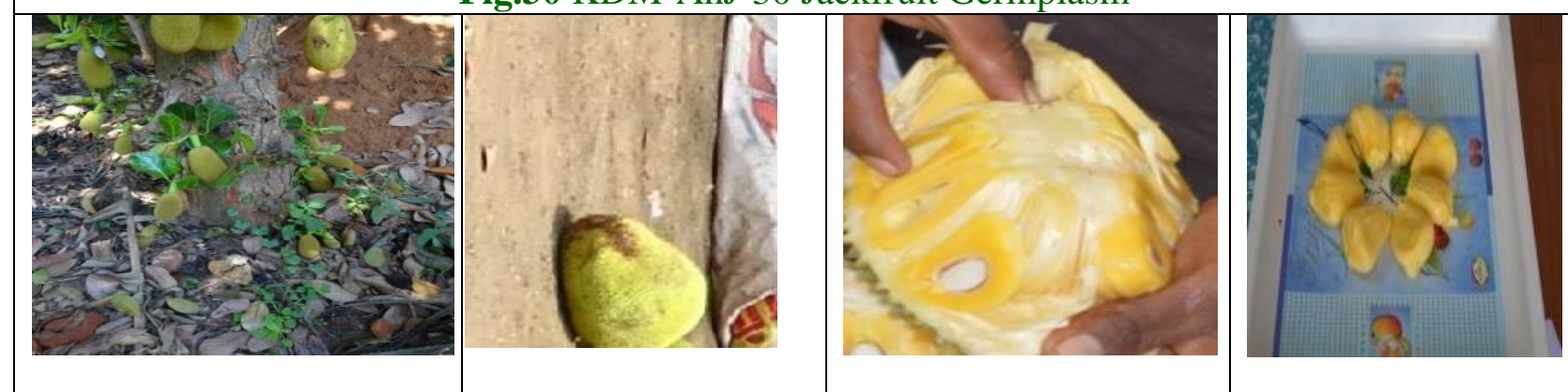

Fig.31 KDM-AhJ-31 Jackfruit Germplasm
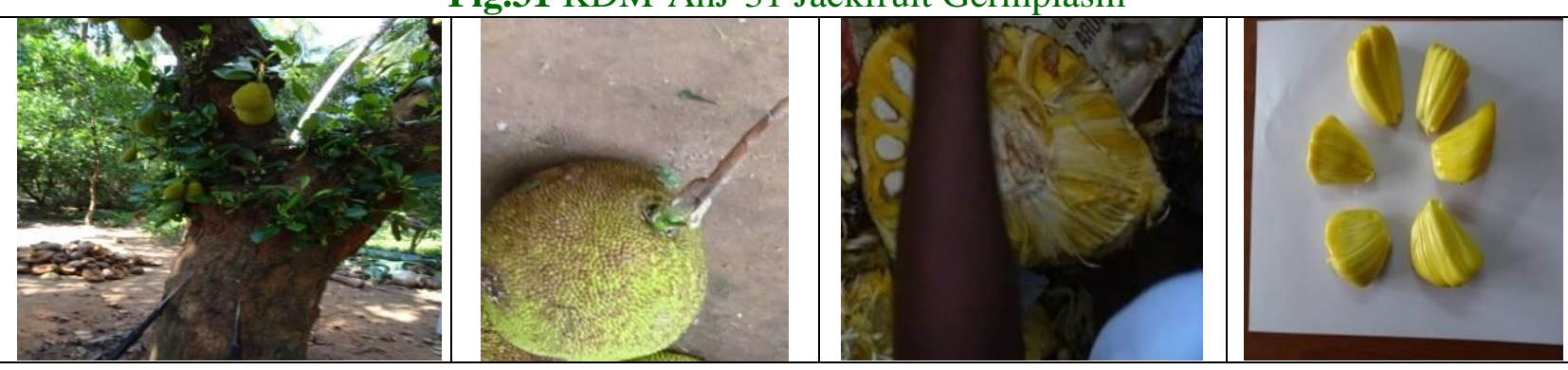

Fig.32 KDM-AhJ-32 Jackfruit Germplasm

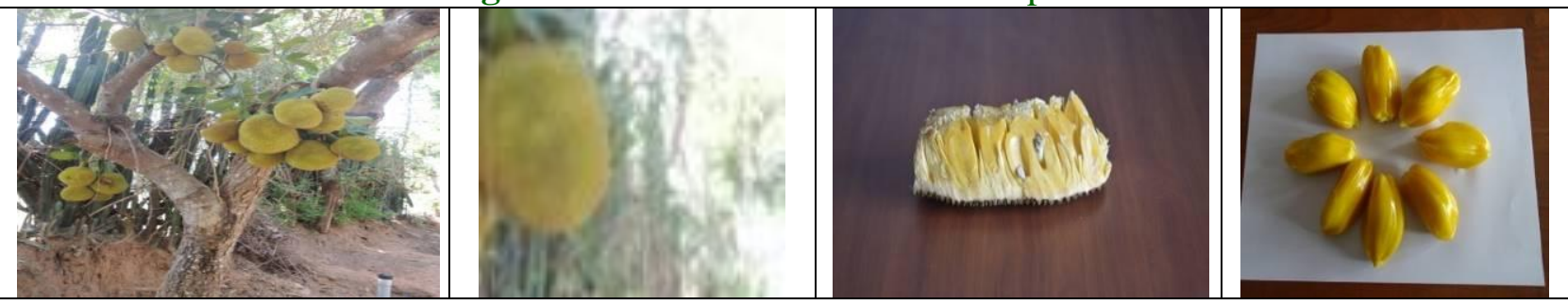

Fig.33 KDM-AhJ-33 Jackfruit Germplasm
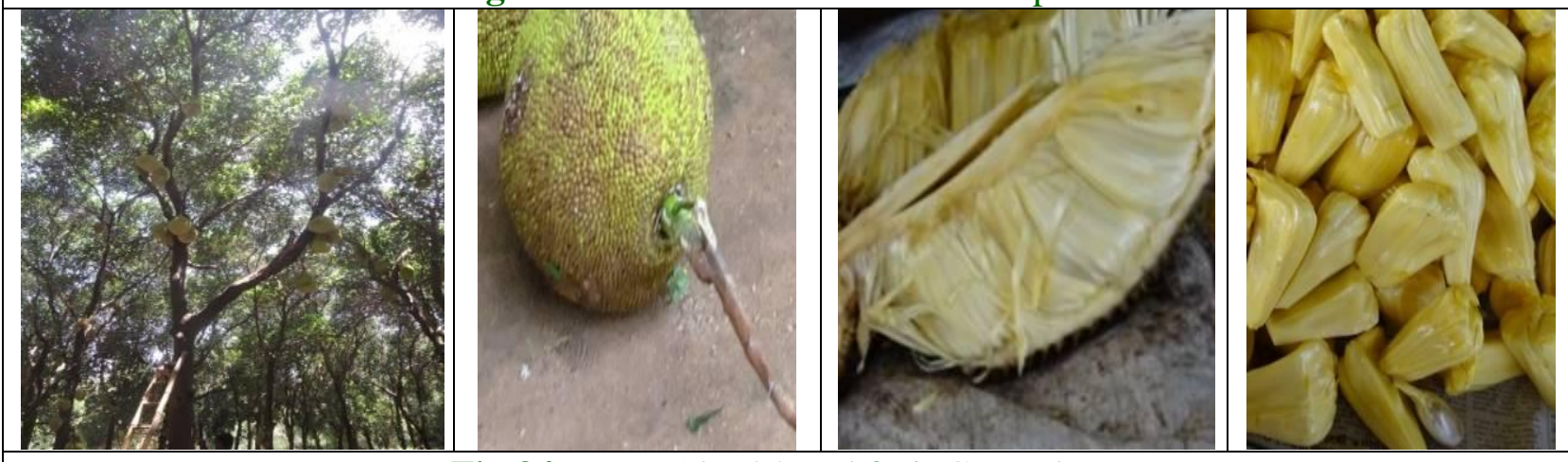

Fig.34 KDM-AhJ-34 Jackfruit Germplasm 


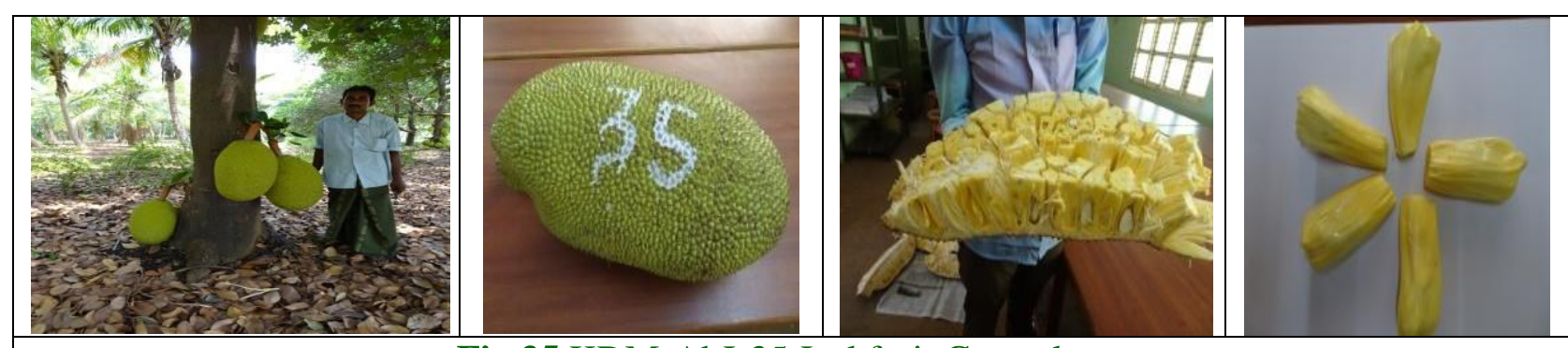

Fig.35 KDM-AhJ-35 Jackfruit Germplasm

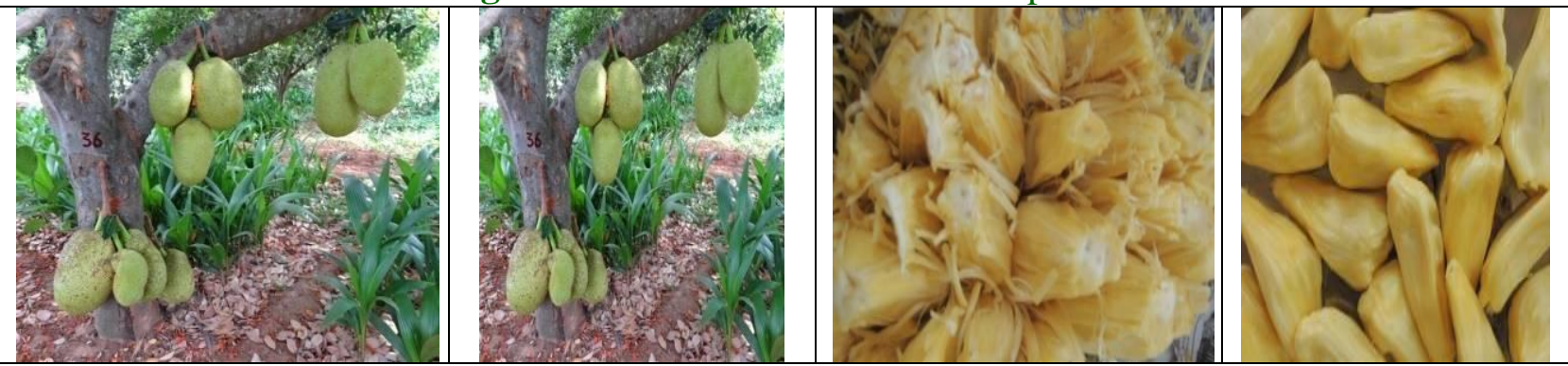

Fig.36 KDM-AhJ-36 Jackfruit Germplasm

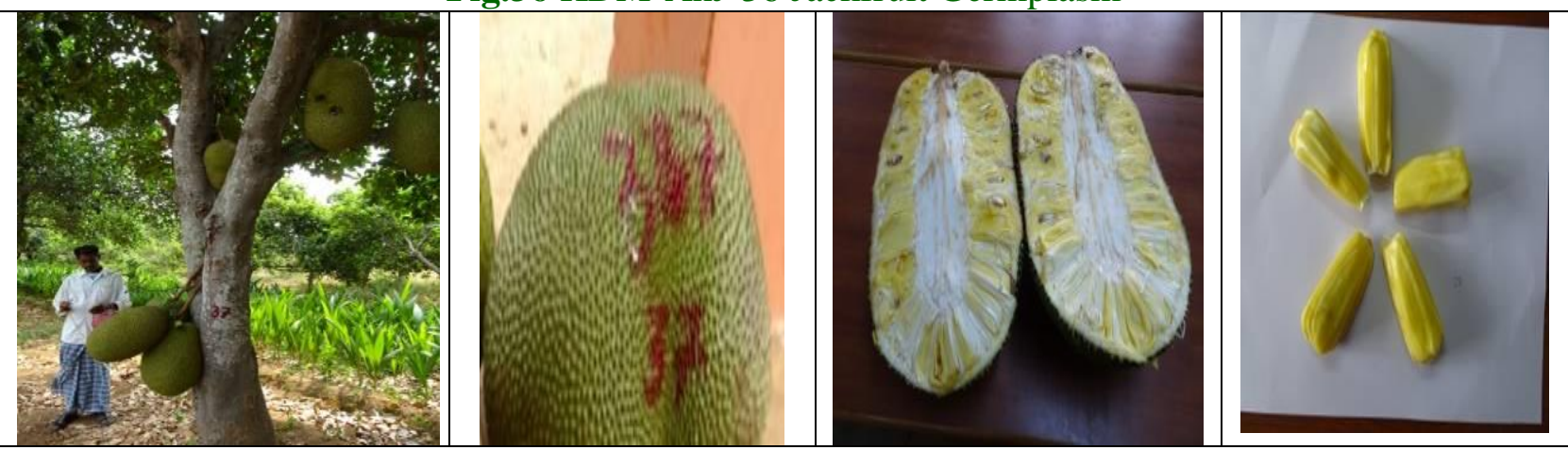

Fig.37 KDM-AhJ-37 Jackfruit Germplasm

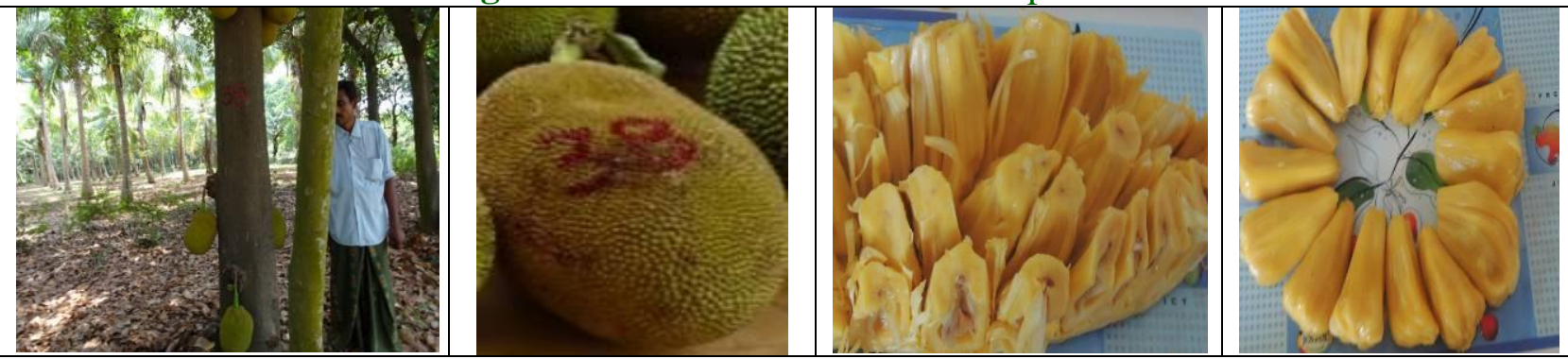

Fig.38 KDM-AhJ-38 Jackfruit Germplasm
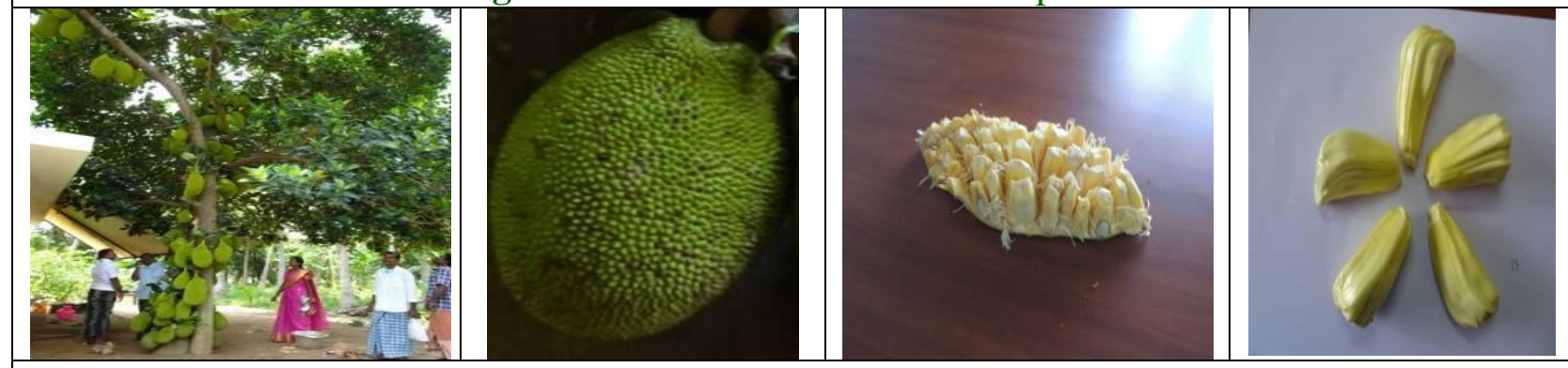

Fig.39 KDM-AhJ-39 Jackfruit Germplasm 

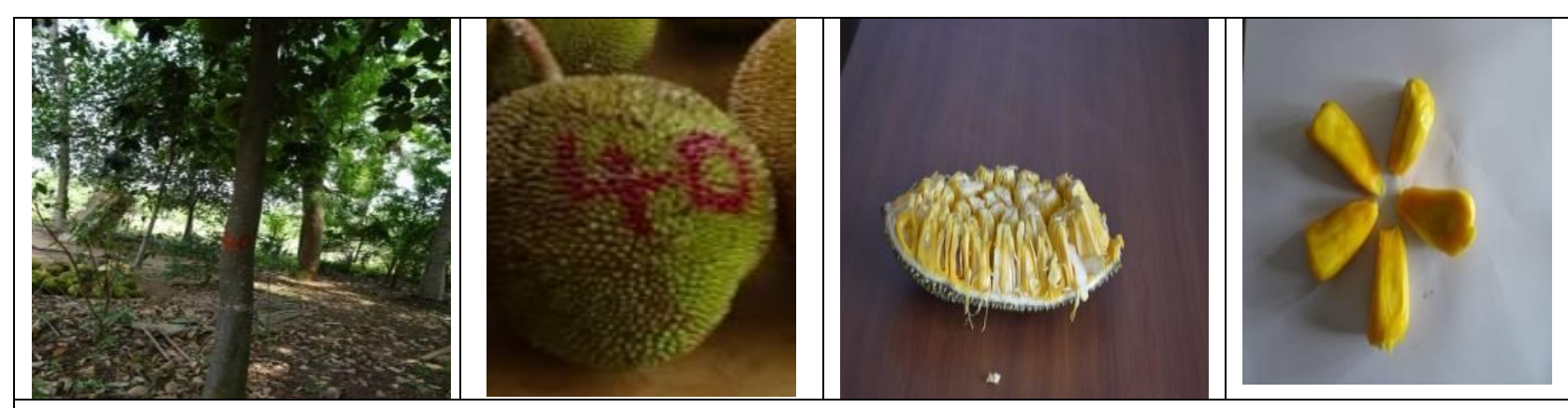

Fig.40 KDM-AhJ-40 Jackfruit Germplasm

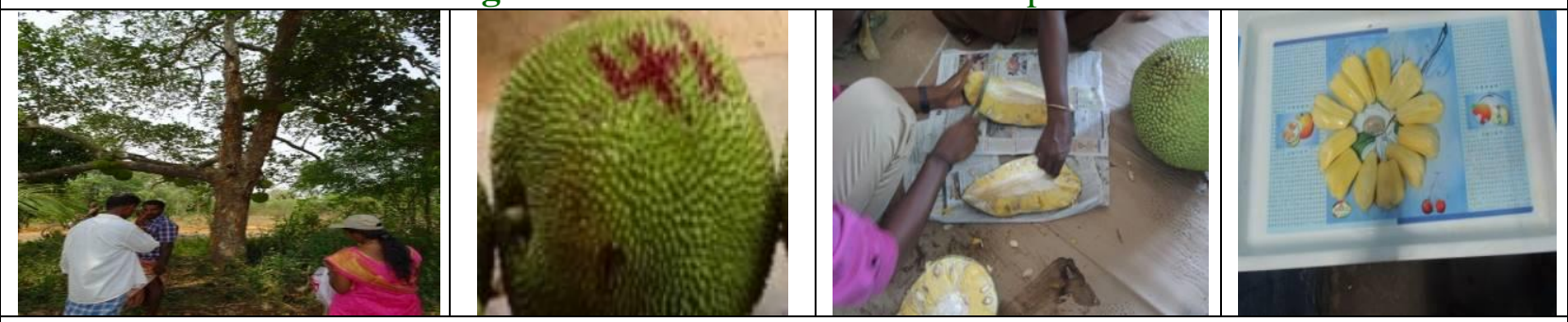

Fig.41 KDM-AhJ-41 Jackfruit Germplasm

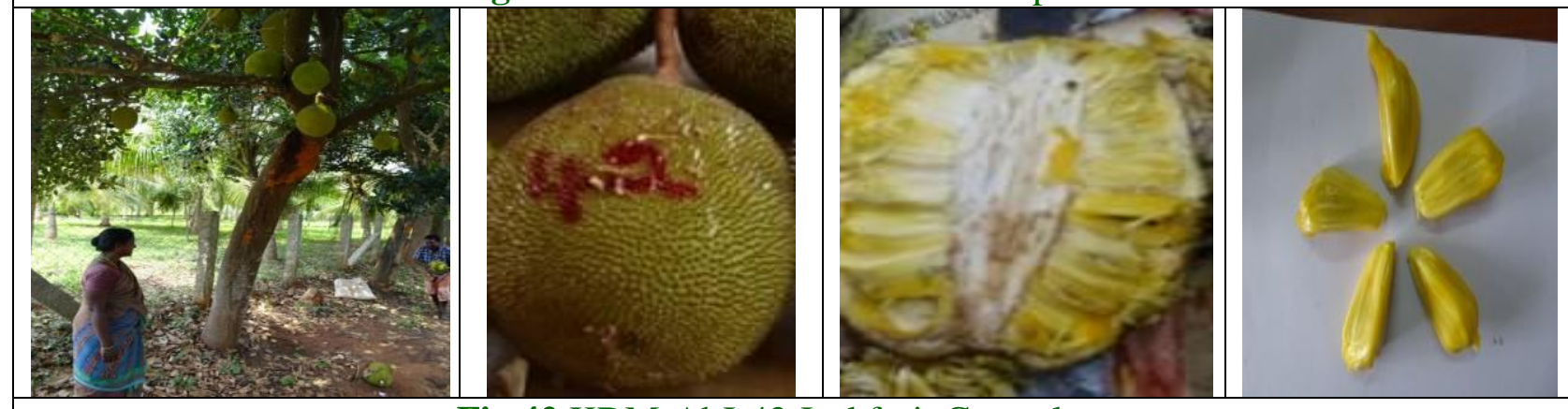

Fig.42 KDM-AhJ-42 Jackfruit Germplasm
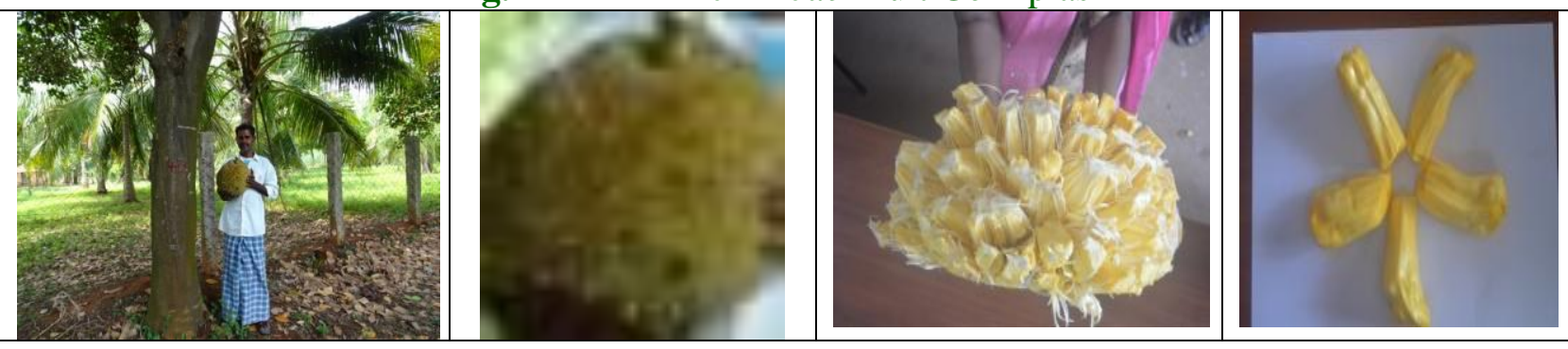

Fig.43 KDM-AhJ-43 Jackfruit Germplasm

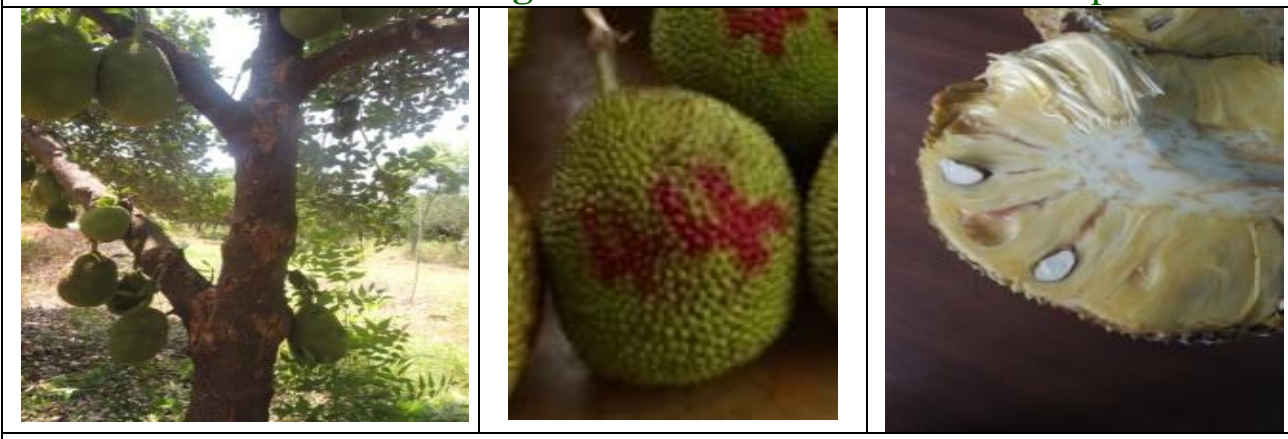

Fig.44 KDM-AhJ-44 Jackfruit Germplasm 

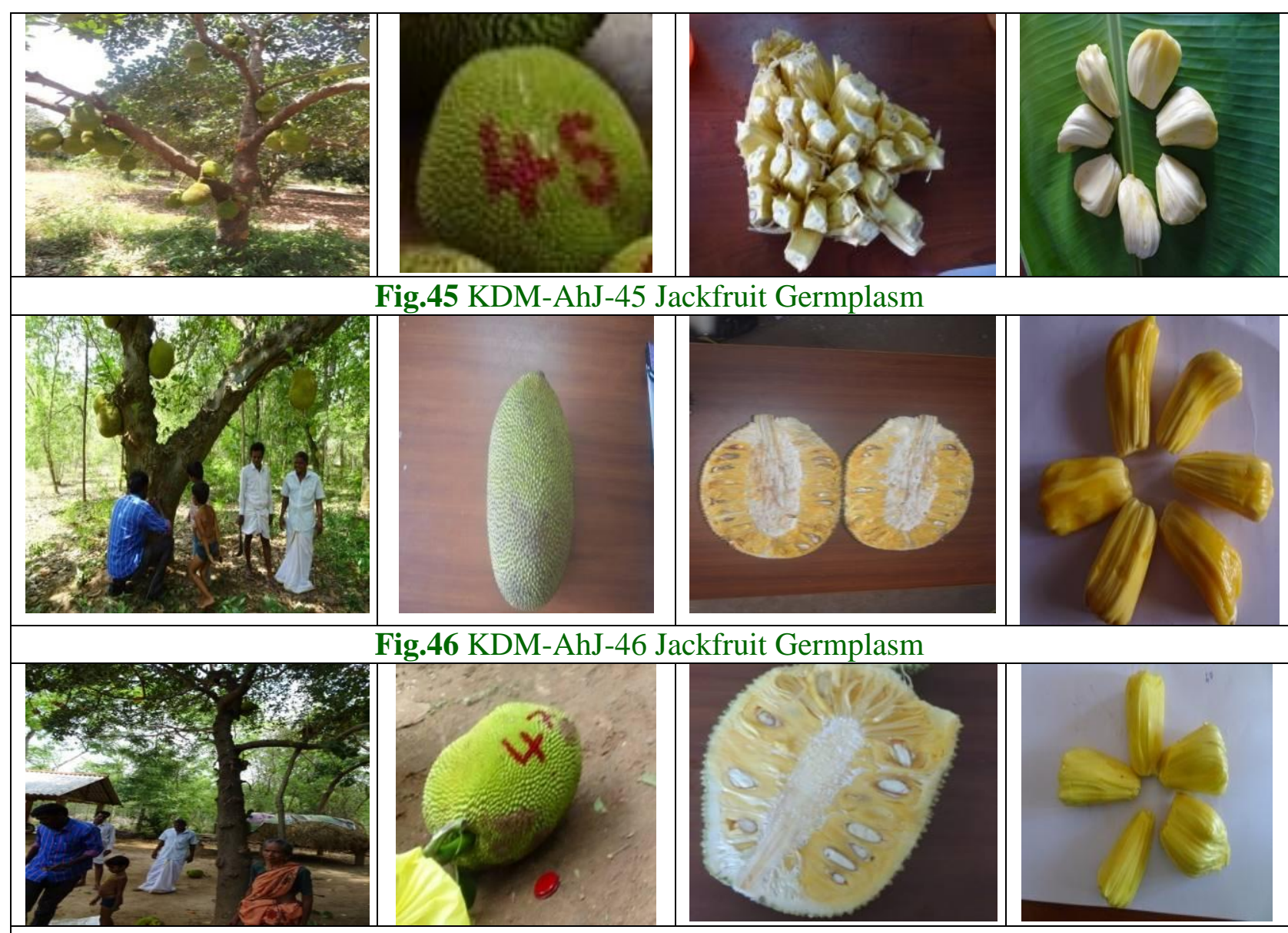

Fig.46 KDM-AhJ-46 Jackfruit Germplasm

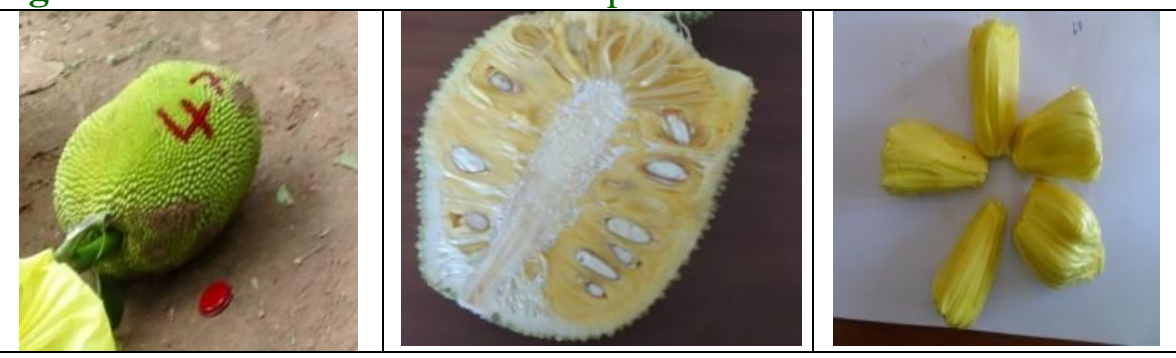

Fig.47 KDM-AhJ-47 Jackfruit Germplasm
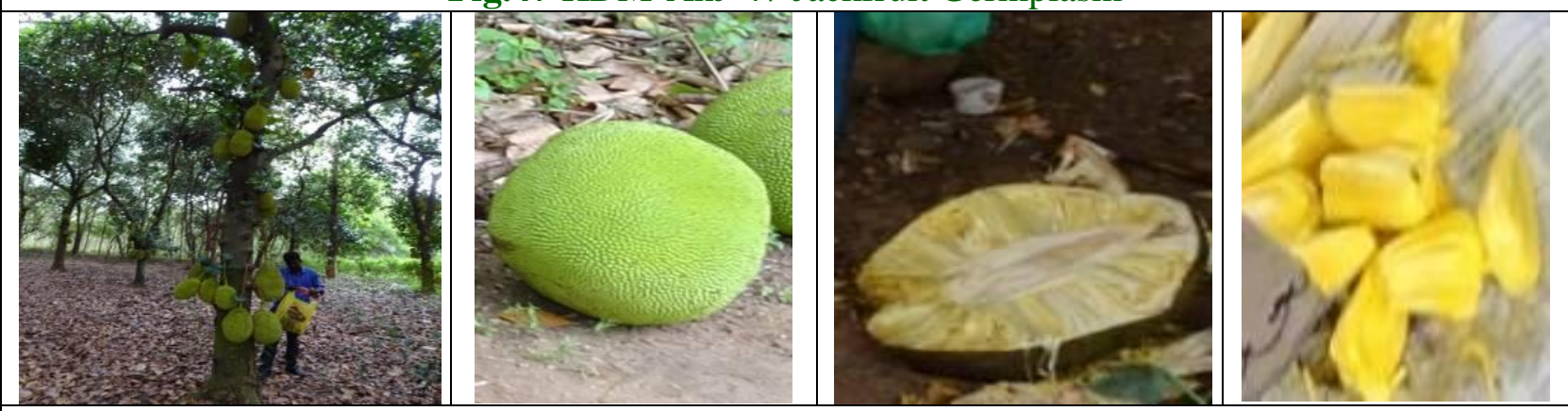

Fig.48 KDM-AhJ-48 Jackfruit Germplasm
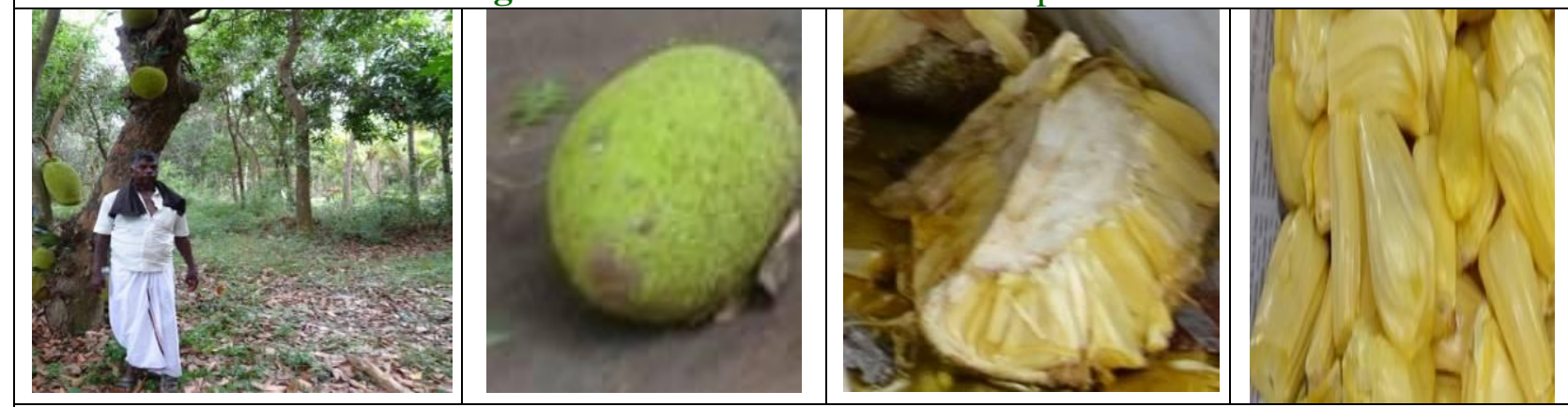

Fig.49 KDM-AhJ-49 Jackfruit Germplasm 


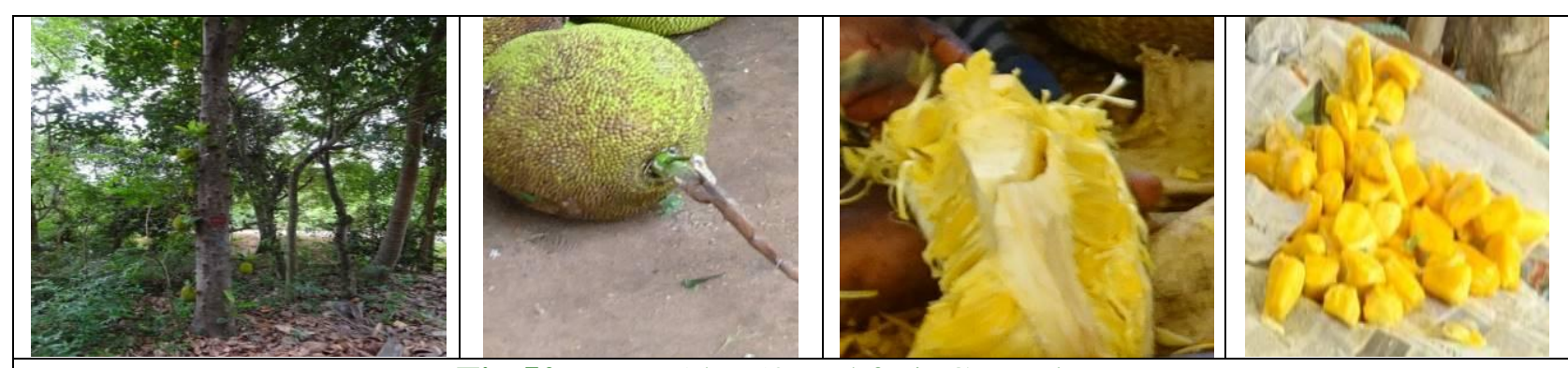

Fig.50 KDM-AhJ-50 Jackfruit Germplasm
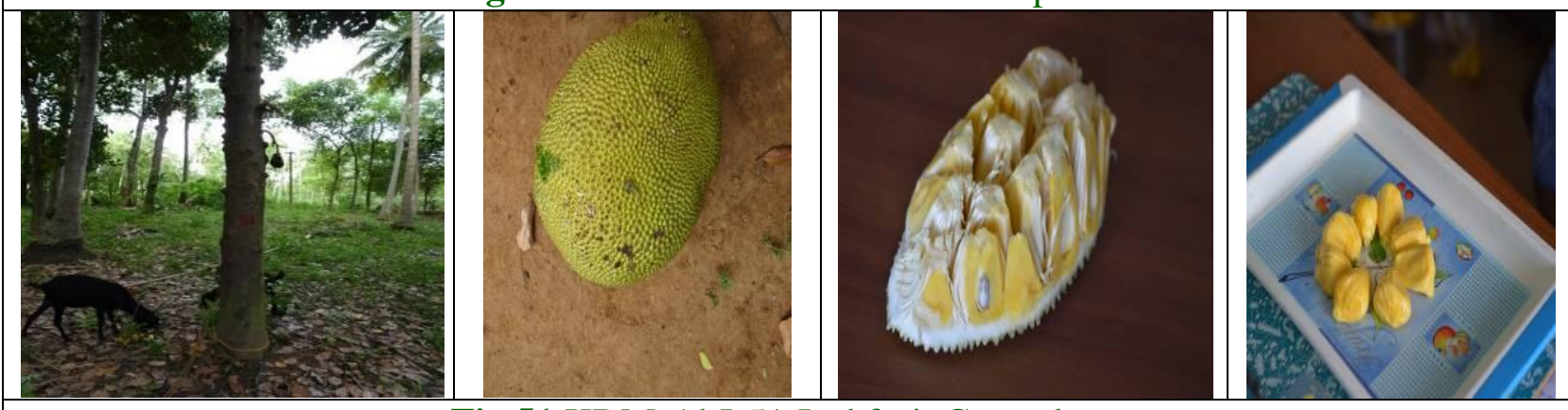

Fig.51 KDM-AhJ-51 Jackfruit Germplasm

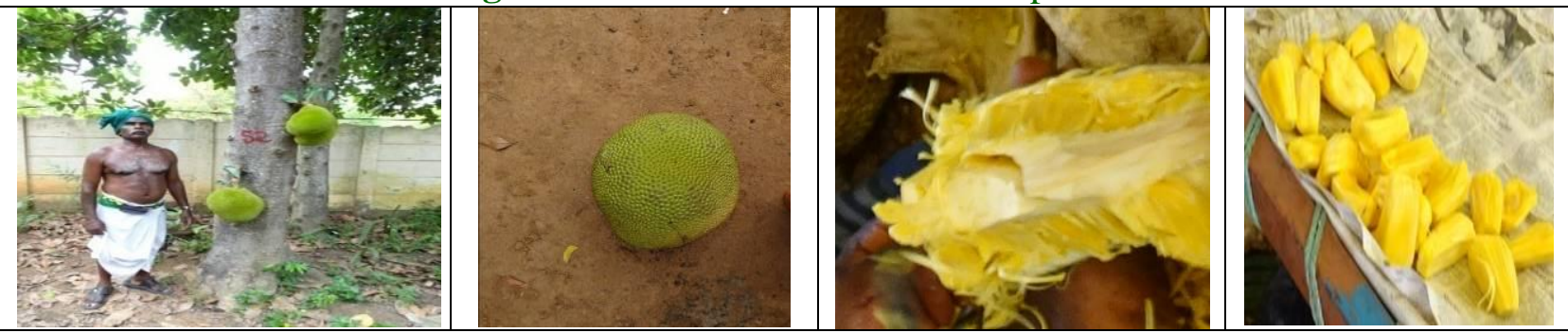

Fig.52 KDM-AhJ-52 Jackfruit Germplasm
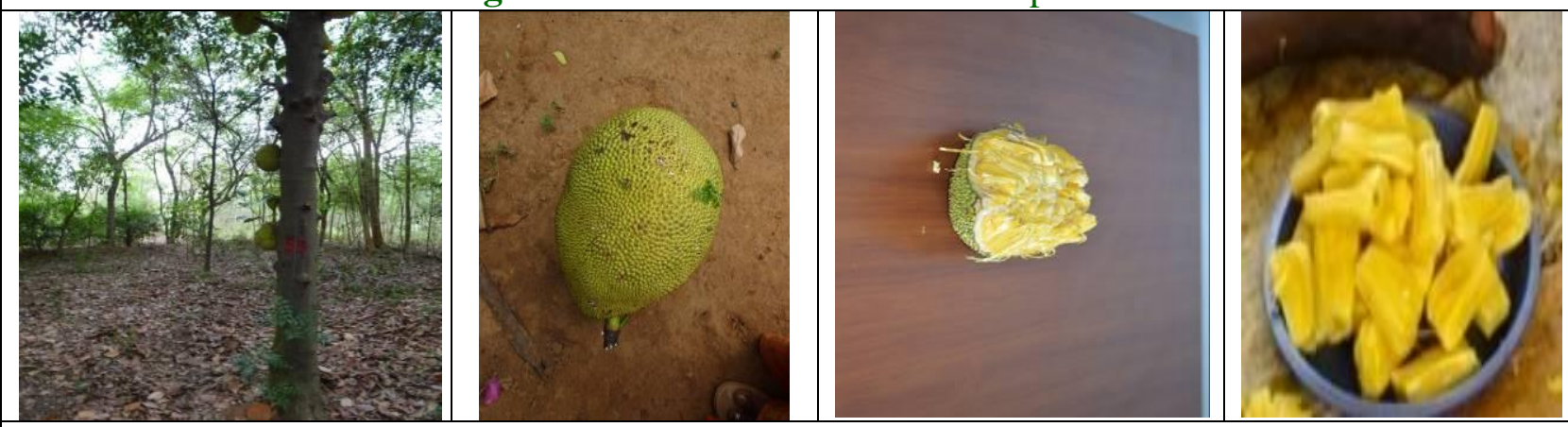

Fig.53 KDM-AhJ-53 Jackfruit Germplasm
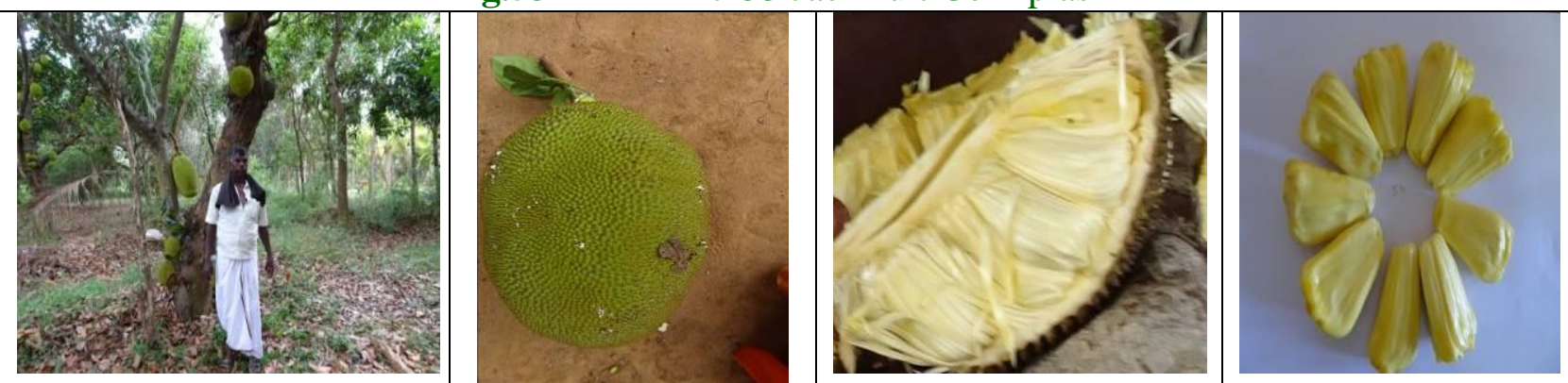

Fig.54 KDM-AhJ-54 Jackfruit Germplasm 


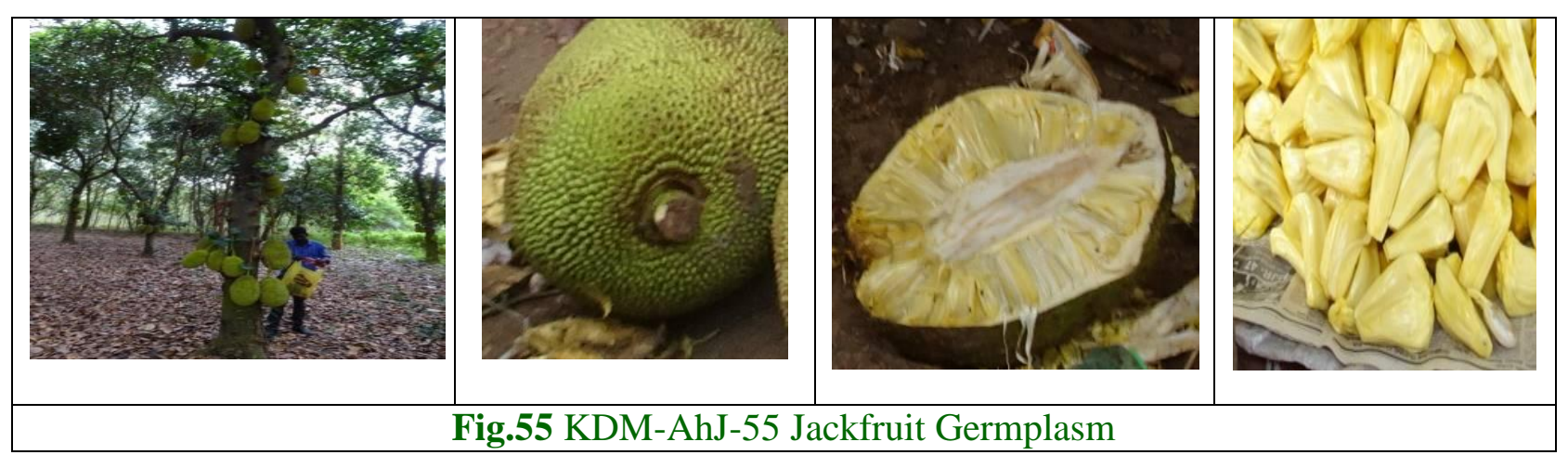

\section{Weather parameter}

Average rainfall during the year 2016 to 2019 was recorded on $465.10 \mathrm{~mm}$ under Pudukkottai District and Alangudi and Thiruvarankulam Taluk of Tamil Nadu (Table 6.).

Maximum temperature of $39^{\circ} \mathrm{C}$ was recorded on during the period of May 2019 and minimum temperature of $25^{\circ} \mathrm{C}$ was recorded on during the December 2018 under Pudukkottai District of Alangudi and Thiruvarankulam Taluk of Tamil Nadu (Table 7.) Jackfruit is a tropical and subtropical fruit. Rainfall and temperature is important factors for initiation flowering and fruiting of jackfruit. Dry tract jackfruits are mostly more sweet compare to irrigated areas. This may be due to the climatic conditions.

Crop improvement is a complex process and these results from the interaction of a combination of factors. Such improvement can be achieved genetically, by developing resistant types, high yield, good quality, fruit characters, (early season, mid-season and late season bearer), by devising improved agronomic practices, by adapting to diverse environments (drought tolerant) and by correcting soil nutrient levels. It can be a major part of any crop improvement programme by the utilization of available genetic resources. An overall improvement strategy of jackfruit (Artocarphus heterophyllus) was taken up by considering the above mentioned justification. Fifty five superior genotypes were selected for environmental adaptation (dry tracts) and high yield, good quality of Jackfruit genotypes. Considering growth, yield and quality characteristics the genotypes KDM-AhJ -08, KDM-AhJ -10 and KDM-AhJ -46 were found to be suitable for jackfruit cultivation at dry tracts of Tamil Nadu.

The present finding shows the great genetic potential of the studied germplasm. It can be used in future breeding programmes for getting productive and quality traits. Moreover, the variability observed in the current study could be used in jackfruit improvement programme. Finally, the plant breeders and horticulturists can choose germplasm according to their needs. Therefore, these jackfruit germplasms can be included in the variety development programme in future.

\section{References}

Akter, H. Rahman.2019. Evaluation of Jackfruit (Artocarpus heterophyllus Lam.) Germplasm. Research \& Reviews: Journal of Botany ISSN: 2278-2222 (Online) Volume 7, Issue1.

Arung ET, Shimizu K and Kondo R (2006). Inhibitory effect of artocarpanone from Artocarpus heterophyllus on melanin biosynthesis. Biol. Pharm. Bull. 29: 19661969.

Azad, A.K., J.G. Jones and N. Haq. 2007. Assessing morphological and isozyme 
variation of jackfruit (Artocarpus heterophyllus Lam.) in Bangladesh. Agroforest Syst., 71(2): 109-125.

Burkill HM (1997). The Useful Plants of West Tropical Africa. 2nd edn. Royal Botanic Gardens, Kew.

Cavagnaro PF, Cavagnaro JB, Lemes JL, Masuelli RW, et al., (2006). Genetic diversity among varieties of the native forage grass Trichloris crinita based on AFLP markers, morphological characters, and quantitative agronomic traits. Genome 49: 906-918.

Craig RE and Harley IM (2006). Artocarpus heterophyllus (jackfruit). Species Profiles for Pacific Island Agroforestry. www.traditionaltree.org. Accessed February 14, 2007.

Department of Statistical, Pudukkottai, 2018.

Hakim EH, Juliawaty LD, Syah YM and Achmad SA (2005). Molecular diversity of Artocarpus champeden (Moraceae): a species endemic to Indonesia. Mol. Divers. 9: 149-158.

Hossain AKMA (1996). Status report on genetic resources of jackfruit in Bangladesh. IPGRI Regional Office, Singapore.

International Plant Genetic Resources Institute. Descriptors for Jackfruit (Artocarpus heterophyllus). Rome: IPGRI; 2000.

IPGRI (2000). Descriptors for jackfruit (Artocarpus heterophyllus Lam.). IPGRI, Rome. ISBN 92-90423-450-3.

Jagadeesh SL, Reddy BS, Basavaraj N, Swamy GSK, et al., (2007). Inter tree variability for fruit quality in jackfruit selections of Western Ghats of India. Sci. Hort. 112: 382-387.

Jagadeesh, S.L., Reddy, B.S., Basavaraj, N., Swamy, G.S.K. and Hegde, L. (2010). Variability studies in physic-chemical qualities of jack fruit (Artocarpus heterophyllus Lam.) of coastal zone of Karnataka. Karnataka J. Agric. Sci., 23 : 293-297.

Murugan, P. 2007. Variability in jack (Artocarpus heterophyllus Lam.). M.Sc., (Hort.) Thesis submitted to Horticultural
College and Research Institute, TNAU, Periyakulam.

Muthulakshmi, P. 2003. Genetic diversity and canopy Management in jackfruit (Artocarpus heterophyllus Lam.). Ph.D., (Hort.) Thesis submitted to Kerala Agricultural University, Thrissur.

Odoemelam SA (2005). Functional properties of raw and heat processed jackfruit (Artocarpus heterophyllus) flour. Pak. J. Nutr. 4: 366-370.

Rahman AKMM, Huq E, Mian AJ and Chesson A (1995). Microscopic and chemical changes occurring during the ripening of two forms of jackfruit (Artocarpus heterophyllus L). Food Chem. 52: 405-410.

Rahman AKMM, Nahar N, Mian AJ and Mosihuzzaman M (1999). Variation of carbohydrate composition of two forms of fruit from jack tree (Artocarpus heterophyllus L) with maturity and climatic conditions. Food Chem. 65: 91-97.

Rowe-Dutton P (1985). Artocarpus heterophyllus - jackfruit. In: The propagation of tropical fruit trees (Garner RJ and Chaudhri SA, eds.). FAO, Rome (Italy); Commonwealth Bureau of Horticulture and Plantation Crops, Maidstone, 269-290.

Saha MC, Saha MG, Rahman MA, Nazrul MI, et al., (1996). Variability in jackfruit. A paper presented at the Internal Research Review Workshop. Horticulture Research Centre, BARI, Gazipur, Bangladesh, 1-4.

Sammadar HM (1985). Jackfruit. In: Fruits of India: Tropical and Subtropical (Bose TK and Mitra SK, eds.). Naya Prokash, Calcutta, 638-649.

Singh A (1986). Fruit Physiology and Production. Kalyani Publishers, New Delhi-Ludhiana, 349-367.

The Hindu. (2015). Mumbai market extends sweet welcome to Vadakadu Jackfruits.

Vinning $G$ and Moody T (1997). A Market Compendium of Tropical Fruits. RIRDC Research Report No. 97/74. Rural Industries Res. and Development Corporation, Barten. 


\section{How to cite this article:}

Jayavalli. R. 2020. Identification and Evaluation of High Yielding Good Quality Jack Genotypes. Int.J.Curr.Microbiol.App.Sci. 9(06): 2429-2456.

doi: https://doi.org/10.20546/ijcmas.2020.906.298 